\title{
High-fidelity state transfer between leaky quantum memories
}

\author{
Daniel Soh $\odot,{ }^{1, *}$ Eric Chatterjee, ${ }^{1, *}$ and Matt Eichenfield ${ }^{2}$ \\ ${ }^{1}$ Sandia National Laboratories, Livermore, California 94550, USA \\ ${ }^{2}$ Sandia National Laboratories, Albuquerque, New Mexico 87123, USA
}

(Received 23 November 2020; revised 28 April 2021; accepted 4 June 2021; published 6 July 2021)

\begin{abstract}
Employing the scattering-Lindblad-Hamiltonian formalism description of quantum network theory, we model the general problem of quantum state transfer between two disparate quantum memory blocks in an open quantum system. We derive an analytical expression for the fidelity of quantum state transfer between the memory blocks under the action of a specific phase space trajectory for each of the relevant classical control fields. We find a set of trajectories that maximize the state transfer fidelity between asymmetric systems. We show that, for the example where the mechanical modes of two optomechanical oscillators act as the quantum memory blocks, their optical modes and a waveguide channel connecting them can be used to achieve a quantum state transfer fidelity of $96 \%$ with realistic parameters using our optimal control solution. The effects of the intrinsic losses and the asymmetries in the physical memory parameters are discussed quantitatively.
\end{abstract}

DOI: 10.1103/PhysRevResearch.3.033027

\section{INTRODUCTION}

Many hybrid quantum systems are being explored to enhance the functionality, scalability, and resource-constrained processing power of near-term quantum information processing systems and networks [1-4]. High-fidelity quantum state transfer will enable key network functions such as entanglement distribution [5] and quantum repeaters [6]. It also allows for various distributed quantum information processing architectures [7,8]. Quantum memories with long decoherence time serve an important role in these systems by reducing the error correction burden associated with decoherence and loss $[9,10]$, as well as by providing coherent quantum storage for processes that require asynchronous qubit operations in algorithms [11]. Thus an ideal quantum memory is a physical system with a long decoherence time and read and write functions enabled via a controllable coupling to intermediary qubits that can propagate between processing blocks and memory blocks or between memory blocks. Regardless of the physical instantiation of quantum memory architecture, the crucial problem to solve is how to configure the time-varying coupling between the storage and intermediary qubits to accomplish optimal quantum state transfer between memory blocks and other elements.

In recent years, there has been significant progress in understanding how to transfer quantum information to different nodes of a quantum network. For example, Cirac et al. considered quantum state transfer between two atomic quantum memories coupled by a photonic channel and optical control fields. They showed that this transfer could be made highly

\footnotetext{
*These authors contributed equally to this work.

Published by the American Physical Society under the terms of the Creative Commons Attribution 4.0 International license. Further distribution of this work must maintain attribution to the author(s) and the published article's title, journal citation, and DOI.
}

efficient by using a complex but realizable pulse modulation scheme [5]. Since then, various experiments have demonstrated parts or all of this scheme on optical, microwave, and atomic systems, such as the experimental demonstration over a photonic channel [12] and reversible state transfer between light and an atom [13]. Some studies have considered the quantum state transfer between flying photons and cavity storage quanta [14]. More recently, microwave photon quantum state transfer was experimentally demonstrated [15,16], along with teleportation between optical beams and mechanical modes [17]. However, the analysis so far does not have the capacity to describe the transfer in the presence of losses and nonidealities, such as leakage into undesired channels. Particularly, an optimal strategy for quantum state transfer between two asymmetric quantum systems with differing energies between the two qubit basis states is completely missing. In a realistic and scalable quantum network, it is highly expected that quantum information will be transferred between nodes that are asymmetric due to the conflicting requirements for performing various quantum operations in each node.

Here, we present the generalized optimal quantum state transfer scheme for two asymmetric quantum systems. Our theory covers quantum memory blocks composed of both identical physical systems and distinct physical systems (with different energy gaps between the corresponding two qubit basis states). Our theory is applicable to both the fermionic memory states (such as spin states) and bosonic memory states (such as phonons). We develop a general model of quantum state transfer for open quantum systems with controllable coupling rates and derive a completely general analytical solution to the optimal control trajectories that accomplishes the highest quantum fidelity obtainable. Knowing the optimal quantum state transfer scheme is crucial to realizing a practical and truly scalable quantum network or hybrid quantum computer. For this, we adopt the emerging scatteringLindblad-Hamiltonian (SLH) formalism description of quantum network theory $[18,19]$ to model the entire system and 
derive the relevant system evolution. Based on the fullquantum treatment, we demonstrate that one can determine the optimal quantum state transfer strategy for given system parameters. Our treatment includes realistic nonidealitiesi.e., the intrinsic losses-and shows that the optimal solution and the final quantum state transfer fidelity are functions of the intrinsic losses. As a concrete example, we will consider two disparate optomechanical quantum memory blocks for optimal quantum state transfer. It will be shown that employing the mechanical mode of an optomechanical oscillator as a quantum memory is tremendously advantageous when implementing such a scheme, since it provides a versatile and controllable coupling mechanism via photon field, along with the desired long decoherence time via the phonon field [20,21].

We emphasize that the previous result obtained by Cirac et al. [5] considered only fully symmetric systems where the derivations are significantly simpler than our current results. A legitimate question then is why there has not been a follow-up result for the asymmetric systems given the long time gap between the result of Cirac et al. and our result. It is because solving the quantum state transfer between asymmetric systems is not a straightforward expansion of the result given by Cirac et al. . Any attempt at such a rudimentary expansion based on the approach of Cirac et al. would fail. We overcame this enormous challenge by way of using the new Hilbert spaces and corresponding operators for dark and bright modes that capture the asymmetric nature completely in the defined operators. This method led to affine quantum master equations and produced an exact solution to the problem of asymmetric systems. We further show that applying the result given by Cirac et al. directly to an asymmetric system case produces a significantly poorer fidelity compared with the one from our derived formula. This proves that our derived formula for general asymmetric systems is a substantial advancement.

The main goal of this paper is to obtain the optimal time-varying control trajectories that accomplish the highest quantum transfer fidelity. For this, we start with a quantum state transfer model in Sec. II, where the SLH formalism will be used to derive the time evolution of qubit states. Based on this full-quantum model, Sec. III solves for the optimal control trajectories for given system parameters, and Sec. IV provides useful numerical examples with realistic optomechanical cavity parameters.

\section{MODEL}

In general, it is useful to build quantum memory from long-decoherence quanta (e.g., phonons), while employing quanta with high propagation velocities (e.g., photons) to transfer information between memory blocks. As such, our goal is to determine how the coupling coefficient between each memory state and the traveling intermediate quantum states connecting the two blocks can be modulated over time such that the transduction fidelity is maximized from the first block to the second block via the intermediate states. Figure 1 depicts the quantum state transfer between two generic quantum memories in block 1 (S1) and block 2 (S2). The aim is to transfer the quantum information initially stored in the long-decoherence memory state of block 1 (S1) to the long-decoherence memory state of block 2 (S2).

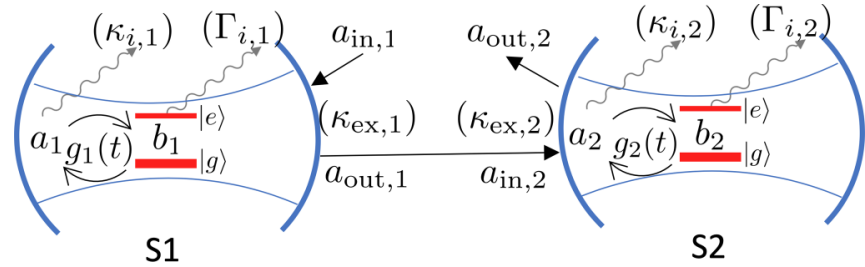

FIG. 1. A schematic for general quantum state transfer between two disparate generic memory blocks. $\left[b_{1}, b_{2}\right.$, annihilation operators for the long-decoherence memory states; $a_{1}, a_{2}$, annihilation operators for the intermediate states that couple with the input and the output of the memory blocks; $g_{1}(t), g_{2}(t)$, the adjustable coupling between the memory states and the intermediate states; $\Gamma_{i, 1}, \Gamma_{i, 2}$, the intrinsic loss rates of the memory states; $\kappa_{i, 1}, \kappa_{i, 2}$, the intrinsic loss rates of the intermediate states].

As for the stored quantum information, we will assume a qubit composed of the two basis states $|g\rangle$ and $|e\rangle$, representing vacuum and singly excited states, respectively. Intermediate quantum states in memory blocks, which couple with the input and the output of the memory blocks, mediate the quantum information to and from the memory states when the coupling mechanism $\left[g_{1}(t), g_{2}(t)\right]$ is turned on.

To start the analysis, we establish a Hilbert space composed of a tensor product of the memory state for S1, the memory state for $\mathrm{S} 2$, the intermediate quantum state for $\mathrm{S} 1$, and the intermediate quantum state for $\mathrm{S} 2$, represented by the annihilation operators $b_{1}, b_{2}, a_{1}, a_{2}$ and creation operators $b_{1}^{\dagger}, b_{2}^{\dagger}$, $a_{1}^{\dagger}, a_{2}^{\dagger}$, respectively. The $\mathrm{S} 1$ memory is initially in an arbitrary superposition of ground and excited states, corresponding to the coefficients $c_{g}$ and $c_{e}$. Therefore the initial state of the overall system can be expressed as follows:

$$
\begin{aligned}
\left|\Psi\left(t_{i}\right)\right\rangle & =\left(c_{g}|g\rangle+c_{e}|e\rangle\right)|g\rangle|00\rangle \\
& =c_{g}|g g\rangle|00\rangle+c_{e}|e g\rangle|00\rangle .
\end{aligned}
$$

The two slots of the first ket are the memory states of S1 and S2, respectively, and those of the second ket are the intermediate states of $\mathrm{S} 1$ and $\mathrm{S} 2$. Here, we assume that the memory states are qubits. Our goal is to faithfully transmit the excitation in $b_{1}$ to $b_{2}$, such that the final state becomes

$$
\left|\Psi\left(t_{f}\right)\right\rangle=c_{g}|g g\rangle|00\rangle+c_{e}|g e\rangle|00\rangle .
$$

Note that as long as the system starts with 0 or 1 excitation in the $\mathrm{S} 1$ memory mode and vacuum in all other modes, energy conservation ensures that the total number of excitations in the system at any given time can never exceed 1 , thus restricting the Hilbert space to five dimensions, spanned by $|g g\rangle|00\rangle$, $|e g\rangle|00\rangle,|g g\rangle|10\rangle,|g g\rangle|01\rangle$, and $|g e\rangle|00\rangle$. This ensures that the probability that the $b_{2}$ mode will be multiply occupied at the end of the transfer process is always zero. Furthermore, the fact that the total excitation number is capped at 1 also makes our model valid for both bosonic modes and two-level fermionic or fermionlike modes (for example, Cirac et al. [5] use a two-level atom for each memory mode).

Next, we determine the Hamiltonian governing this system. Our system consists of two blocks $i=1,2$, with the following self-energy Hamiltonian for each:

$$
H_{\mathrm{self}}^{(i)}=\hbar \omega_{c}\left(a_{i}^{\dagger} a_{i}+b_{i}^{\dagger} b_{i}\right)
$$


Note that we have engineered the system such that the memory state and the intermediate quantum state for each block are resonant with each other. This accords with the treatment by Cirac et al. [5] and is justified by the need to maximize the quantum state transfer efficiency. Specifically, detuning between the states would sharply attenuate the effective interaction rate and thus massively slow down the quantum state transfer. Furthermore, also in accordance with Cirac et al., we engineer the S1 and S2 modes to be resonant with each other. This is also due to the need to maximize the transfer efficiency, since any detuning between the intermediate quantum state of S1 and that of S2 would sharply hinder absorption of the output of S1 into S2.

Given a time-varying interaction $g(t)$ between the memory state and the intermediate quantum state, we consider a class of memory blocks that couples the mediating cavity field and the long-term memory field via the following beam-splitter interaction Hamiltonian, per Cirac et al. [5]:

$$
H_{\mathrm{int}}^{(i)}=-\hbar g_{i}(t)\left(a_{i}^{\dagger} b_{i}+a_{i} b_{i}^{\dagger}\right) .
$$

We note that this form for the interaction Hamiltonian applies to all systems for which the interaction processes conserve the total number of quanta. In fact, this model covers a vast majority of quantum memory configurations since in most quantum memories the read and write function is accomplished via such a beam-splitter coupling Hamiltonian between the mediating cavity field (photons or phonons) and the long-term memory state. We note that this model covers both the direct coupling, such as dipole coupling between a cavity photon field and the spin memory state, and the parametric coupling between the photon field and the phonon memory states in an optomechanical system. In addition, practical quantum memories featuring beam-splitter atom-photon interaction have already been realized [22,23]. For the example of optomechanical oscillators as the memory block, $g(t)=g_{0} \sqrt{\bar{n}(t)}$ with the single-photon coupling coefficient $g_{0}=G x_{\text {zpf }} . G=-\partial \omega_{\text {cav }} / \partial x$ is the slope of the photonic resonance frequency over the displacement $x$ of the mechanical system, and $x_{\mathrm{zpf}}$ is the zero-point-fluctuation parameter of the mechanical system. Varying the control $g(t)$ is accomplished through varying the photon population $\bar{n}(t)$ at a red-detuned driving frequency, while varying $g_{0}$ is also possible as we will explain later. For another example of the neutral atoms as the memory block, $g(t)$ is the Rabi oscillation frequency, a function of dipole moment and an adjustable driving electromagnetic field amplitude.

Adding the self-energy and the interaction Hamiltonian yields the following total Hamiltonian for each block in the static frame:

$$
\begin{aligned}
H^{(i)} & =H_{\mathrm{self}}^{(i)}+H_{\mathrm{int}}^{(i)} \\
& =\hbar \omega_{c}\left(a_{i}^{\dagger} a_{i}+b_{i}^{\dagger} b_{i}\right)-\hbar g_{i}(t)\left(a_{i}^{\dagger} b_{i}+a_{i} b_{i}^{\dagger}\right) .
\end{aligned}
$$

Due to the resonance among the modes, it is convenient to express it in the rotating frame, which leaves only the interaction terms:

$$
H^{(i)}=-\hbar g_{i}(t)\left(a_{i}^{\dagger} b_{i}+a_{i} b_{i}^{\dagger}\right) .
$$

Next, it is essential to consider the various loss channels from these blocks, which we will model using Lindblad operators.
In particular, the state transfer process utilizes the output channel of the first memory block to connect to the second memory block's input channel. We will label the rates for this coherent extrinsic loss process as $\kappa_{\mathrm{ex}, 1}$ and $\kappa_{\mathrm{ex}, 2}$ for $\mathrm{S} 1$ and $\mathrm{S} 2$, respectively. In addition, we will need to account for the incoherent intrinsic losses from the blocks. For the respective blocks, we label the loss rates for the intermediate quanta as $\kappa_{i, 1}$ and $\kappa_{i, 2}$ and the decay rates for the memory storage quanta as $\Gamma_{i, 1}$ and $\Gamma_{i, 2}$. The Lindblad operators for the two blocks are thus expressed in the following manner:

$$
L_{1}=\left(\begin{array}{c}
\sqrt{\kappa_{\mathrm{ex}, 1}} a_{1} \\
\sqrt{\kappa_{i, 1}} a_{1} \\
\sqrt{\Gamma_{i, 1}} b_{1}
\end{array}\right), \quad L_{2}=\left(\begin{array}{c}
\sqrt{\kappa_{\mathrm{ex}, 2}} a_{2} \\
\sqrt{\kappa_{i, 2}} a_{2} \\
\sqrt{\Gamma_{i, 2}} b_{2}
\end{array}\right) .
$$

We now consider the overall system consisting of the two blocks. Per Gardiner and Collett [24], for a mode $n$ coupled to a bath via a single input-output channel, the relationship between the mode annihilation operator $a_{n}$, output annihilation operator $a_{\mathrm{out}, n}$, and input annihilation operator $a_{\mathrm{in}, n}$ is given as follows:

$$
a_{\mathrm{out}, n}=a_{\mathrm{in}, n}+\sqrt{\kappa_{\mathrm{ex}, n}} a_{n},
$$

where $n=1,2$. The input to the intermediate quantum state in the second block is identical to the output of that in the first block, except for an appropriate time delay. One can eliminate the time delay effectively from all formulas by introducing the time-delayed operators for the operators in S2 [5]. Applying this time adjustment, one can set $a_{\mathrm{in}, 2}(t)=a_{\mathrm{out}, 1}(t)$ with the implicit understanding of $a_{\mathrm{in}, 2}(t) \rightarrow \tilde{a}_{\mathrm{in}, 2}(t)\left[=a_{\mathrm{in}, 2}(t-\tau)\right]$, where $\tau$ is the time delay. The output operator for S2, representing a loss channel for the system, then takes the form of a superposition of the two intermediate state annihilation operators and the bath input operator for S1:

$$
a_{\mathrm{out}, 2}=\sqrt{\kappa_{\mathrm{ex}, 1}} a_{1}+\sqrt{\kappa_{\mathrm{ex}, 2}} a_{2}+a_{\mathrm{in}, 1} .
$$

We assume that the bath temperature is sufficiently low and thus the input fields are in vacuum states. Then, $a_{\text {in,1 }}$ returns a null value when applied on the system state. According to the series connection rule of the SLH formalism [18], the connected system's Hamiltonian $H_{T}$ and Lindbladian $L_{T}$ take the following form:

$$
\begin{gathered}
H_{T}=-\hbar g_{1}(t)\left(a_{1}^{\dagger} b_{1}+a_{1} b_{1}^{\dagger}\right)-\hbar g_{2}(t)\left(a_{2}^{\dagger} b_{2}+a_{2} b_{2}^{\dagger}\right) \\
+\frac{i \hbar}{2} \sqrt{\kappa_{\mathrm{ex}, 1} \kappa_{\mathrm{ex}, 2}}\left(a_{1}^{\dagger} a_{2}-a_{1} a_{2}^{\dagger}\right), \\
L_{T}=\left(\begin{array}{c}
\sqrt{\kappa_{i, 1}} a_{1} \\
\sqrt{\Gamma_{i, 1}} b_{1} \\
\sqrt{\kappa_{\mathrm{ex}, 1}} a_{1}+\sqrt{\kappa_{\mathrm{ex}, 2}} a_{2} \\
\sqrt{\kappa_{i, 2}} a_{2} \\
\sqrt{\Gamma_{i, 2}} b_{2}
\end{array}\right) .
\end{gathered}
$$

One way to physically explain the above result is as follows: the subprocesses corresponding to extrinsic loss from the individual blocks are split into a part representing coherent coupling between blocks, which we incorporate in the composite Hamiltonian $H_{T}$, and a part represented by $a_{\text {out,2 }}$ corresponding to system loss due to reflection of the propagating wave packet from the second block, which enters into the Lindbladian $L_{T}$. 


\section{ANALYTICAL SOLUTION}

It is useful to incorporate the Lindblad operators into the Hamiltonian in order to model the nonunitary time evolution of the wave function in the basis of the excited states. In general, for a set of loss channels labeled $n$, the effective Hamiltonian takes the following form [5]:

$$
H^{\mathrm{eff}}=H-\sum_{n} \frac{i}{2} L_{n}^{\dagger} L_{n} .
$$

Since coherent reflection $a_{\text {out, } 2}$ serves as the dominant loss channel, we start by expanding the Hamiltonian to incorporate the corresponding Lindblad operator:

$$
H^{\prime}=H_{T}-\frac{i \hbar}{2}\left(\sqrt{\kappa_{\mathrm{ex}, 1}} a_{1}^{\dagger}+\sqrt{\kappa_{\mathrm{ex}, 2}} a_{2}^{\dagger}\right)\left(\sqrt{\kappa_{\mathrm{ex}, 1}} a_{1}+\sqrt{\kappa_{\mathrm{ex}, 2}} a_{2}\right) .
$$

By default, this effective Hamiltonian is non-Hermitian for the overall Hilbert space. However, we can design the couplings $g_{1}(t)$ and $g_{2}(t)$ such that the system remains in a dark state throughout the transfer process so that the system states evolve coherently [5].

We first ignore the intrinsic losses. Defining the dimensionless constant $\epsilon=\kappa_{\mathrm{ex}, 2} / \kappa_{\mathrm{ex}, 1}$, we derive the dark mode (whose annihilation operator is represented by $a_{d}$ ) by superposing $a_{1}$ and $a_{2}$ for the intermediate modes so that applying the annihilation operator $a_{d}=a_{1}+\sqrt{\epsilon} a_{2}$ onto the dark state returns a null value. To solve for this dark mode, we construct the following generic superposition of $a_{1}$ and $a_{2}$ modes:

$$
\mid \text { dark }\rangle=c_{1}|10\rangle+c_{2}|01\rangle .
$$

Applying $a_{d}$ to this state and setting the output to 0 , we derive the following equation relating $c_{1}$ and $c_{2}$ :

$$
c_{1}+\sqrt{\epsilon} c_{2}=0 .
$$

The coefficients $c_{1}$ and $c_{2}$ must also satisfy the normalization condition:

$$
(1+\epsilon)\left|c_{2}\right|^{2}=1
$$

Solving the above two equations and substituting into Eq. (A2), we find the dark mode wave function in terms of the two cavity modes:

$$
\mid \text { dark }\rangle=\frac{1}{\sqrt{1+\epsilon}}(|01\rangle-\sqrt{\epsilon}|10\rangle) .
$$

We note that this dark state produces zero quanta in the S2 output channel (no information leakage) as the connected system's Lindblad operator produces a null result on this state.

It is also necessary to define the bright mode (whose annihilation operator is represented by $a_{b}$ ) as orthogonal to the dark mode:

$$
\mid \text { bright }\rangle=\frac{1}{\sqrt{1+\epsilon}}(\sqrt{\epsilon}|01\rangle+|10\rangle) .
$$

The bright state produces nonzero $\mathrm{S} 2$ output quanta, losing quantum information from the connected system, and therefore we want to avoid such a bright state in order to maximize the quantum state transfer fidelity.

Next, we construct ladder operators corresponding to the new basis for the intermediate state Hilbert subspace, so that we can then rewrite the Hamiltonian in terms of those operators. We denote the annihilation operators for the dark and bright modes as

$$
\begin{gathered}
a_{d}=|00\rangle\langle\text { dark }|, \\
a_{b}=|00\rangle\langle\text { bright }|,
\end{gathered}
$$

respectively. These ladder operators can be related to $a_{1}$ and $a_{2}$ by applying the required Hilbert space rotation:

$$
\begin{aligned}
& a_{d}=\frac{1}{\sqrt{1+\epsilon}}\left(a_{2}-\sqrt{\epsilon} a_{1}\right), \\
& a_{b}=\frac{1}{\sqrt{1+\epsilon}}\left(\sqrt{\epsilon} a_{2}+a_{1}\right) .
\end{aligned}
$$

The Hamiltonian $H^{\prime}$ is thus expressed in terms of the new intermediate state basis as follows:

$$
\begin{aligned}
H^{\prime}= & \hbar g_{1}(t) \frac{\sqrt{\epsilon}}{\sqrt{1+\epsilon}}\left(a_{d}^{\dagger} b_{1}+a_{d} b_{1}^{\dagger}\right)-\hbar g_{1}(t) \frac{1}{\sqrt{1+\epsilon}}\left(a_{b}^{\dagger} b_{1}+a_{b} b_{1}^{\dagger}\right)-\hbar g_{2}(t) \frac{1}{\sqrt{1+\epsilon}}\left(a_{d}^{\dagger} b_{2}+a_{d} b_{2}^{\dagger}\right) \\
& -\hbar g_{2}(t) \frac{\sqrt{\epsilon}}{\sqrt{1+\epsilon}}\left(a_{b}^{\dagger} b_{2}+a_{b} b_{2}^{\dagger}\right)+i \hbar \frac{\kappa_{\mathrm{ex}, 1}}{2} \sqrt{\epsilon}\left(a_{b}^{\dagger} a_{d}-a_{b} a_{d}^{\dagger}\right)-i \hbar \frac{\kappa_{\mathrm{ex}, 1}}{2}(1+\epsilon) a_{b}^{\dagger} a_{b} .
\end{aligned}
$$

Note that for the kernel of the $a_{b}^{\dagger} a_{b}$ operator (i.e., the reduced Hilbert space where applying $a_{b}^{\dagger} a_{b}$ produces a null result for all elements), $H^{\prime}$ becomes Hermitian because the system does not decohere. Given the initial composite state described in Eq. (1), the time-dependent composite state can be expressed in terms of generic coefficients $\alpha_{1}(t), \alpha_{2}(t)$, and $\beta_{\alpha}(t)$ :

$$
\begin{aligned}
|\Psi(t)\rangle= & c_{g}|g g\rangle|00\rangle+c_{e}\left[\alpha_{1}(t)|e g\rangle|00\rangle\right. \\
& \left.\left.+\alpha_{2}(t)|g e\rangle|00\rangle+i \beta_{\alpha}(t)|g g\rangle \mid \text { dark }\right\rangle\right] .
\end{aligned}
$$

Next, we introduce the intrinsic, decohering loss processes to the effective Hamiltonian. Since we design both blocks such that these intrinsic loss rates are equally minimal, we assume that $\kappa_{i}=\kappa_{i, 1}=\kappa_{i, 2}$ and $\Gamma_{i}=\Gamma_{i, 1}=\Gamma_{i, 2}$ for simplicity. However, we note that this simplifying assumption can be removed, and it is straightforward to derive the general result in the case of asymmetric intrinsic losses. This results in the effective Hamiltonian

$$
H_{T}^{\mathrm{eff}}=H^{\prime}-i \hbar \frac{\kappa_{i}}{2}\left(a_{b}^{\dagger} a_{b}+a_{d}^{\dagger} a_{d}\right)-i \hbar \frac{\Gamma_{i}}{2}\left(b_{1}^{\dagger} b_{1}+b_{2}^{\dagger} b_{2}\right) .
$$

Now that we have decoherence in the system, by breaking the Hermiticity of the Hamiltonian, the intrinsic losses introduce 
nonunitarity to the time evolution in the Hilbert space of the excited states, such that $\left|\alpha_{1}(t)\right|^{2}+\left|\alpha_{2}(t)\right|^{2}+\left|\beta_{\alpha}(t)\right|^{2}<1$ for any time $t>t_{i}$. Conceptually, the decline of the normalization value over time corresponds to the increasing probability that the system has collapsed to the vacuum state $|g g\rangle|00\rangle$, which effectively increases the value of $\left|c_{g}\right|^{2}$.

Applying the Schrödinger equation, we obtain the dynamically coupled differential equations:

$$
\begin{gathered}
\dot{\alpha_{1}}(t)=\frac{\sqrt{\epsilon}}{\sqrt{1+\epsilon}} g_{1}(t) \beta_{\alpha}(t)-\frac{\Gamma_{i}}{2} \alpha_{1}(t), \\
\dot{\alpha}_{2}(t)=-\frac{1}{\sqrt{1+\epsilon}} g_{2}(t) \beta_{\alpha}(t)-\frac{\Gamma_{i}}{2} \alpha_{2}(t), \\
\dot{\beta_{\alpha}}(t)=\frac{1}{\sqrt{1+\epsilon}} g_{2}(t) \alpha_{2}(t)-\frac{\sqrt{\epsilon}}{\sqrt{1+\epsilon}} g_{1}(t) \alpha_{1}(t) \\
-\frac{\kappa_{i}}{2} \beta_{\alpha}(t) .
\end{gathered}
$$

To prevent the system from entering the bright mode, we constrict the range of coupling profiles $g_{1}(t)$ and $g_{2}(t)$ such that

$$
\left\langle g g, \text { bright }\left|H_{T}^{\text {eff }}\right| \Psi(t)\right\rangle=0 .
$$

This yields a fourth equation for the coefficients:

$$
\begin{aligned}
0= & \frac{\sqrt{\epsilon}}{\sqrt{1+\epsilon}} g_{2}(t) \alpha_{2}(t)+\frac{1}{\sqrt{1+\epsilon}} g_{1}(t) \alpha_{1}(t) \\
& +\frac{\kappa_{\mathrm{ex}, 1} \sqrt{\epsilon}}{2} \beta_{\alpha}(t) .
\end{aligned}
$$

Appendixes A-C demonstrate the method for solving the time evolution of the coefficients, defining $t=0$ as the halfway point of the process. To reduce the range of possible coupling profiles, we set $g_{1}(t)$ and $g_{2}(t)$ as constant for $t \geqslant 0$ and $t \leqslant 0$, respectively, following Cirac et al. [5]. The reasoning is that in the second (first) half of the process, the excited population will largely be in $\mathrm{S} 2$ (S1), and therefore changes in the coupling for S1 (S2) will not substantially alter the state populations. It is worth noting that Xu et al. [25] used a linear combination of Gaussian functions to construct the coupling profiles but also obtained an approximately flat shape for the driving field for segment 1 (2) when the excited population was largely in segment 2 (1). For $t \geqslant 0$, we derive the following expressions (see the Appendix B:

$$
\begin{gathered}
\alpha_{1}(t)=\frac{e^{-\frac{1}{4}\left(\kappa_{\mathrm{ex}, 1}+\kappa_{i}+\Gamma_{i}\right) t}}{C}\left[\alpha_{1}(0) C \cosh \left(\frac{C}{4} t\right)+B_{2}^{\prime} \sinh \left(\frac{C}{4} t\right)\right], \\
\beta_{\alpha}(t)=\frac{e^{-\frac{1}{4}\left(\kappa_{\mathrm{ex}, 1}+\kappa_{i}+\Gamma_{i}\right) t}}{C}\left[\beta_{\alpha}(0) C \cosh \left(\frac{C}{4} t\right)-B_{1}^{\prime} \sinh \left(\frac{C}{4} t\right)\right], \\
\alpha_{2}(t)^{2}=\frac{e^{-\frac{1}{2}\left(\kappa_{\mathrm{ex}, 1}+\kappa_{i}+\Gamma_{i}\right) t}}{16 g_{1}(0)^{2}}\left\{\left(\kappa_{\mathrm{ex}, 1} \beta_{\alpha}(0)^{2}+\frac{2 g_{1}(0)}{\sqrt{\epsilon(1+\epsilon)}} \alpha_{1}(0) \beta_{\alpha}(0)\right) A_{1}(t)+\left[2 \kappa_{\mathrm{ex}, 1} B_{1}^{\prime} \beta_{\alpha}(0)\right.\right. \\
\left.\left.+\frac{2 g_{1}(0)}{\sqrt{\epsilon(1+\epsilon)}}\left(\alpha_{1}(0) B_{1}^{\prime}-\beta_{\alpha}(0) B_{2}^{\prime}\right)\right] A_{2}(t)+\left(\kappa_{\mathrm{ex}, 1} B_{1}^{\prime 2}-\frac{2 g_{1}(0)}{\sqrt{\epsilon(1+\epsilon)}} B_{1}^{\prime} B_{2}^{\prime}\right) A_{3}(t)\right\}+G e^{-\Gamma_{i} t} .
\end{gathered}
$$

Here, $C=\sqrt{\left(\Gamma_{i}-\kappa_{\mathrm{ex}, 1}-\kappa_{i}\right)^{2}-16 g_{1}(0)^{2}}$. Note that as $t$ increases, $\alpha_{1}(t)$ and $\beta_{\alpha}(t)$ converge to zero as expected, since the system should eventually either transition fully to the $b_{2}$ memory mode or decay to the vacuum state. The functions $A_{1}(t), A_{2}(t)$, and $A_{3}(t)$ are defined as

$$
\begin{gathered}
A_{1,3}(t)=\frac{\mp C^{2}-C\left(\Gamma_{i}-\kappa_{i}-\kappa_{\mathrm{ex}, 1}\right) \sinh \left(\frac{C}{2} t\right)+\left(\Gamma_{i}-\kappa_{i}-\kappa_{\mathrm{ex}, 1}\right)^{2}\left[\cosh \left(\frac{C}{2} t\right) \pm 1\right]}{C^{1 \mp 1}\left(\Gamma_{i}-\kappa_{i}-\kappa_{\mathrm{ex}, 1}\right)} \\
A_{2}(t)=\frac{-\left(\Gamma_{i}-\kappa_{i}-\kappa_{\mathrm{ex}, 1}\right) \sinh \left(\frac{C}{2} t\right)+C \cosh \left(\frac{C}{2} t\right)}{C}
\end{gathered}
$$

and the constants $B_{1}^{\prime}$ and $B_{2}^{\prime}$ are

$$
\begin{aligned}
& B_{1}^{\prime}=4 \sqrt{\frac{1+\epsilon}{\epsilon}} g_{1}(0) \alpha_{1}(0)+\beta_{\alpha}(0)\left(\kappa_{\mathrm{ex}, 1}+\kappa_{i}-\Gamma_{i}\right), \\
& B_{2}^{\prime}=4 \sqrt{\frac{\epsilon}{1+\epsilon}} g_{1}(0) \beta_{\alpha}(0)+\alpha_{1}(0)\left(\kappa_{\mathrm{ex}, 1}+\kappa_{i}-\Gamma_{i}\right) .
\end{aligned}
$$

Regarding the term $G e^{-\Gamma_{i} t}$, it is useful to note that $\Gamma_{i} \ll$ $\kappa_{\mathrm{ex}, 1}, \kappa_{i}$ due to the extremely long memory decoherence time. Therefore, over the timescale of the transfer process, $e^{-\Gamma_{i} t}$ will approximately equal 1 , resulting in $G \approx \alpha_{2}^{2}\left(t_{f}\right)$, which represents the fidelity of quantum state transfer.
For $t \leqslant 0$, the coefficient solutions take a similar form (see Appendix C), with the replacements $\alpha_{1} \leftrightarrow \alpha_{2}, \quad \kappa_{\mathrm{ex}, 1} \rightarrow-\epsilon \kappa_{\mathrm{ex}, 1}, \quad C \rightarrow D^{\prime} \quad$ [where $\quad D^{\prime}=$ $\left.\sqrt{\left(\Gamma_{i}+\epsilon \kappa_{\mathrm{ex}, 1}-\kappa_{i}\right)^{2}-16 g_{2}(0)^{2}}\right], \quad 2 g_{1}(0) / \sqrt{\epsilon(1+\epsilon)} \rightarrow$ $-2 \epsilon g_{2}(0) / \sqrt{1+\epsilon}$ in the coefficient for $\alpha_{1}(t)^{2}, G \rightarrow G^{\prime}$, as well as $B_{1}^{\prime} \rightarrow B_{3}^{\prime}$ and $B_{2}^{\prime} \rightarrow B_{4}^{\prime}$, where

$$
\begin{gathered}
B_{3}^{\prime}=-4(\sqrt{1+\epsilon}) g_{2}(0) \alpha_{2}(0)+\beta_{\alpha}(0)\left(-\epsilon \kappa_{\mathrm{ex}, 1}+\kappa_{i}-\Gamma_{i}\right), \\
B_{4}^{\prime}=-\frac{4 g_{2}(0)}{\sqrt{1+\epsilon}} \beta_{\alpha}(0)+\alpha_{2}(0)\left(-\epsilon \kappa_{\mathrm{ex}, 1}+\kappa_{i}-\Gamma_{i}\right) .
\end{gathered}
$$


TABLE I. Optimal coupling $g_{1}(t)$ and $g_{2}(t)$ for $t \leqslant 0$ and $t \geqslant 0$ producing the highest quantum state transfer fidelity attainable.

\begin{tabular}{lcr}
\hline \hline & $t \leqslant 0$ & $t \geqslant 0$ \\
\hline$g_{1}(t)$ & $-\frac{1}{\alpha_{1}(t)}\left(\frac{\kappa_{\mathrm{ex}, 1} \sqrt{\epsilon(1+\epsilon)}}{2} \beta_{\alpha}(t)+\sqrt{\epsilon} g_{2}(0) \alpha_{2}(t)\right)$ & $g_{1}(0)$ \\
$g_{2}(t)$ & $g_{2}(0)$ & $-\frac{1}{\alpha_{2}(t)}\left(\frac{\kappa_{\mathrm{ex}, 1} \sqrt{1+\epsilon}}{2} \beta_{\alpha}(t)+\frac{1}{\sqrt{\epsilon}} g_{1}(0) \alpha_{1}(t)\right)$ \\
\hline \hline
\end{tabular}

As previously mentioned, $e^{-\Gamma_{i} t} \approx 1$ for all times $t_{i} \leqslant t \leqslant t_{f}$. Therefore $G^{\prime} \approx \alpha_{1}\left(t_{i}\right)^{2}$, and since the system starts out in the $b_{1}$ memory mode, this implies that $G^{\prime} \approx 1$.

Having found solutions for the time evolution of the coefficients $\alpha_{1}(t), \alpha_{2}(t)$, and $\beta_{\alpha}(t)$ in terms of their values at $t=0$, we can now use these functions along with the known constants $g_{1}(0)$ and $g_{2}(0)$ to determine the optimal coupling profile functions. The expressions for $g_{1}(t)$ and $g_{2}(t)$ are shown in Table I (for a detailed derivation, see Appendix D).

\section{NUMERICAL METHODS}

In order to determine numerical solutions for the coupling profiles, we used MATLAB-based computation to calculate the valid sets of $\left(\alpha_{1}(0), \alpha_{2}(0), \beta_{\alpha}(0)\right)$. Due to the extremely long memory decoherence time, we can generally set $\Gamma_{i} \approx 0$. However, the intrinsic intermediate state loss will measurably degrade the fidelity, as depicted in Fig. 2. The inverse correlation between the fidelity and the ratio of intrinsic loss to output coupling rate can be interpreted as follows: The output coupling corresponds to the rate of transfer from S1 to S2. A higher rate shortens the time over which the excitation is in the intermediate form, thus reducing the probability that the excitation decays due to intrinsic loss.

It is also useful to consider the effect of asymmetry between the blocks. Figure 3 depicts the optimal fidelity as a function of the output coupling rates of the two blocks, with the intrinsic loss rates set at the minimal value, i.e., $\kappa_{i}=\kappa_{i, 1}=\kappa_{i, 2}$. As expected, the fidelity declines if either of the extrinsic loss rates is reduced, approximately fitting an exponential profile, which follows from the nearly exponential variation of the fidelity with the reciprocal of the extrinsic loss as shown in Fig. 2.

As a useful example, we consider empirical values for optomechanical oscillator blocks. A high-quality

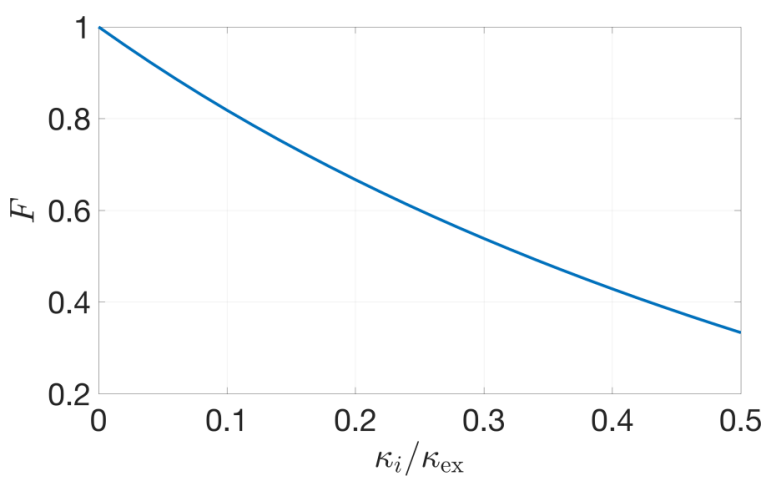

FIG. 2. Optimal fidelity as a function of the ratio of intrinsic loss rate to output coupling rate, assuming equal output coupling rates for the two blocks, i.e., $\kappa_{\mathrm{ex}}=\kappa_{\mathrm{ex}, 1}=\kappa_{\mathrm{ex}, 2}$. optomechanical oscillator features a mechanical damping rate of $\Gamma_{i} \lesssim 2 \pi \times 5 \mathrm{kHz}$ and an intrinsic photon loss rate of $\kappa_{i} \approx$ $2 \pi \times 100 \mathrm{MHz}$, along with an upper bound of $2 \pi \times 5 \mathrm{GHz}$ for the output coupling rates $\kappa_{\mathrm{ex}, 1}$ and $\kappa_{\mathrm{ex}, 2}$. The very low value for the mechanical damping rate meets our requirement that the memory decoherence be negligible over the timescale of the process, and we therefore set $\Gamma_{i} \approx 0$. Having shown that the fidelity is highest for maximal output coupling, we also set $\kappa_{\mathrm{ex}, 1}=2 \pi \times 5 \mathrm{GHz}=\kappa_{\mathrm{ex}, 2}$. For these inputs, our calculations yield an optimal fidelity of about $96 \%$. For the choice of $g_{1}(0)=\kappa_{\mathrm{ex}, 1}=g_{2}(0), \alpha_{1}(0)=0.7, \alpha_{2}(0)=-0.7$, and $\beta_{\alpha}(0)=0$, Fig. 4 depicts the time evolution of the state populations $\left|\alpha_{1}(t)\right|^{2},\left|\alpha_{2}(t)\right|^{2}$, and $\left|\beta_{\alpha}(t)\right|^{2}$ and the corresponding pulse profiles. Note that the total population degrades due to intrinsic loss, with the fidelity represented by the final population $\left|\alpha_{2}\left(t_{f}\right)\right|^{2} \approx 0.96$. For the given $t=0$ values, it is also evident that in the first half of the process, half of the population is transferred to the final phonon mode, which is then repeated with the other half of the population. This indicates that the process is close to time reversible (though full time reversibility is prevented by intrinsic loss), corresponding to the observation that $g_{1}(t)$ and $g_{2}(-t)$ closely match each other.

Next, we consider an example of asymmetric memory blocks, i.e., two blocks with differing output coupling rates. Here, we set $\kappa_{\mathrm{ex}, 1}=2 \pi \mathrm{GHz}$ and $\kappa_{\mathrm{ex}, 2}=2 \pi \times 5 \mathrm{GHz}$, corresponding to an asymmetry factor $\epsilon=5$. Given these inputs, we find an optimal fidelity of $89.1 \%$. Figure 5 depicts the time evolution of the state populations and corresponding pulse profiles for the choice of $g_{1}(0)=\kappa_{\mathrm{ex}, 1}=g_{2}(0), \alpha_{1}(0)=$ $0.6535, \alpha_{2}(0)=-0.6560$, and $\beta_{\alpha}(0)=0.2970$.

It is worth comparing our fidelity result for the case of asymmetric memory blocks with the result that would be predicted based on Cirac et al. [5]. Since Cirac et al. specifically assume two blocks with equal output coupling rates, we apply

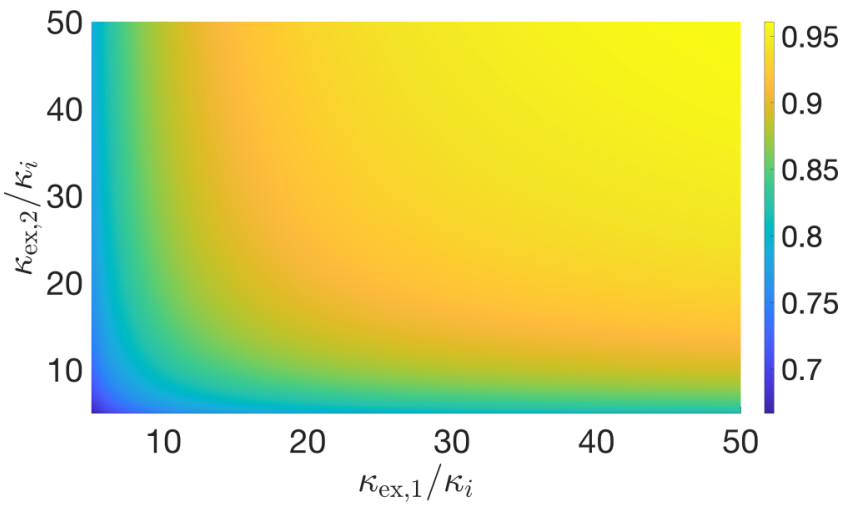

FIG. 3. Optimal fidelity as a function of output coupling to intrinsic loss ratio for $\mathrm{S} 1$ and $\mathrm{S} 2$. 


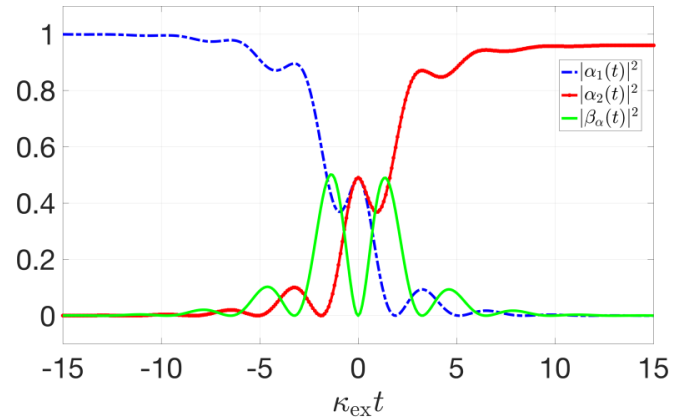

(a)

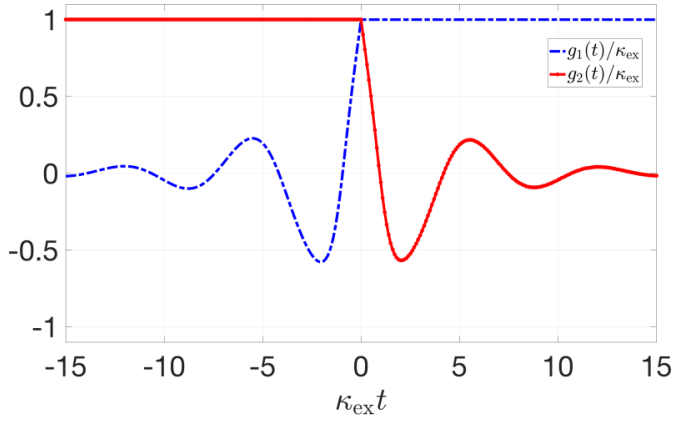

(b)

FIG. 4. Evolution of the state populations $\left|\alpha_{1}(t)\right|^{2},\left|\alpha_{2}(t)\right|^{2}$, and $\left|\beta_{\alpha}(t)\right|^{2}$ (a) and optimal pulse profiles $g_{1}(t)$ and $g_{2}(t)$ (b) over time for $\kappa_{\mathrm{ex}}=\kappa_{\mathrm{ex}, 1}=\kappa_{\mathrm{ex}, 2}=2 \pi \times 5 \mathrm{GHz}, \kappa_{i}=2 \pi \times 100 \mathrm{MHz}, g_{1}(0)=\kappa_{\mathrm{ex}}=g_{2}(0), \alpha_{1}(0)=0.7, \alpha_{2}(0)=-0.7$, and $\beta_{\alpha}(0)=0$.

their theory to asymmetric blocks by rudimentarily averaging the rates, deriving the pulse shapes using this averaged value as the universal output coupling rate, and then applying these pulse shapes to the actual Hamiltonian featuring the asymmetric blocks. Intuitively, we would expect that this would significantly degrade the fidelity, since the dark mode condition is broken and substantial extra loss is incurred due to reflection of the signal traveling from block 1 to block 2 . This is confirmed by examining the case of $\kappa_{\mathrm{ex}, 1}=2 \pi \mathrm{GHz}$ and $\kappa_{\mathrm{ex}, 2}=2 \pi \times 5 \mathrm{GHz}$ discussed above. If the pulse shapes are solved for by setting the output coupling rate for both blocks to the average value of $\kappa_{\mathrm{ex}}=2 \pi \times 3 \mathrm{GHz}$, then a numerical simulation based on Quantum Toolbox in PYTHON (QUTIP [26]) reveals that the maximum fidelity becomes $73.5 \%$, well below the $89.1 \%$ fidelity based on the pulse shapes derived using our method. This example clearly shows that our derivation for the asymmetric systems is substantially critical in real practical systems. Also, it shows that the earlier result by Cirac et al. [5] is not directly applicable to the important examples of asymmetrical systems.

One issue to be addressed is the physically valid range of $g_{1}(t)$ and $g_{2}(t)$. For the example of optomechanical oscillator blocks, if we store the phonon memory in a membrane at an antinode of the cavity wave and then displace the equilibrium position using two laser drives highly detuned from the cavity field, then the coupling coefficient will carry different signs for opposite displacements [27,28]. For generic mem- ory blocks, if we set $\left|g_{1}(0)\right|<\left|\kappa_{\mathrm{ex}, 1}+\kappa_{i}\right| / 4$ and $\left|g_{2}(0)\right|<$ $\left|\epsilon \kappa_{\mathrm{ex}, 1}-\kappa_{i}\right| / 4$, then the constants $C$ and $D^{\prime}$ in the coefficient expressions become real, thereby replacing the oscillatory behavior of the coupling profiles with a superposition of exponentials. In that case, we can maintain fully positive values for $g_{1}(t)$ and $g_{2}(t)$ for select values of $\alpha_{1}(0), \alpha_{2}(0)$, and $\beta_{\alpha}(0)$. Figure 6 depicts the time evolution of the state populations and pulse shapes for the previously discussed case of symmetric memory blocks $\left(\kappa_{\mathrm{ex}, 1}=2 \pi \times 5 \mathrm{GHz}=\kappa_{\mathrm{ex}, 2}\right)$ given the parameters $g_{1}(0)=0.2 \kappa_{\mathrm{ex}, 1}=g_{2}(0), \alpha_{1}(0)=0.7435, \alpha_{2}(0)=$ 0.5470 , and $\beta_{\alpha}(0)=-0.3650$. Here, we see that the Rabi oscillations have disappeared, and $g_{1}(t)$ and $g_{2}(t)$ asymptotically decay to 0 .

We also made an argument earlier that since the system starts with 0 or 1 excitation, the total number of excitations in the system is never greater than 1, which enabled us to restrict the Hilbert space to just the vacuum state and the singly excited states. Figure 7, based on a full-quantum simulation of the density matrix evolution performed in QUTIP, numerically corroborates this analysis, as the final occupation probability for the states $|2\rangle$ and $|3\rangle$ in the $b_{2}$ mode is zero. Furthermore, the simulations confirm the $96 \%$ maximal fidelity value calculated for the optomechanical oscillator, since a transfer process that starts at $|1\rangle$ in the $b_{1}$ mode ends with an occupation probability of 0.96 for $|1\rangle$ in the $b_{2}$ mode, with a probability of 0.04 that the system collapses to vacuum due to the loss channels.

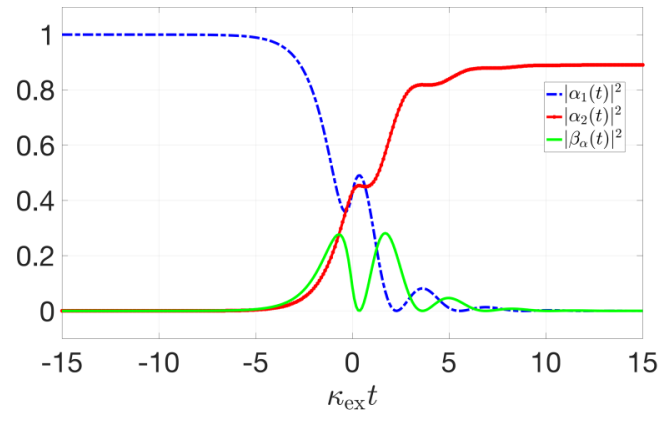

(a)

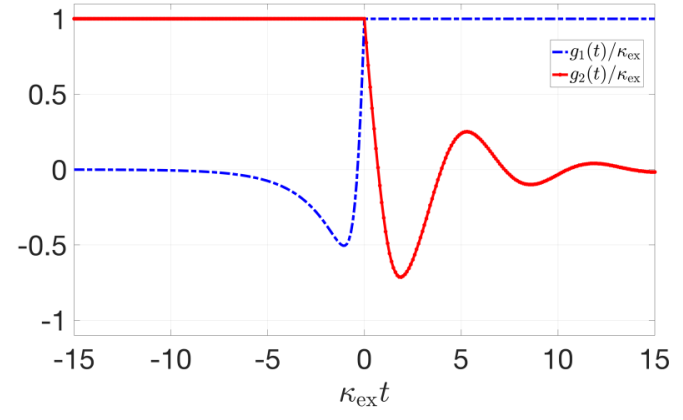

(b)

FIG. 5. Evolution of the state populations $\left|\alpha_{1}(t)\right|^{2},\left|\alpha_{2}(t)\right|^{2}$, and $\left|\beta_{\alpha}(t)\right|^{2}$ (a) and optimal pulse profiles $g_{1}(t)$ and $g_{2}(t)$ (b) over time for $\kappa_{\mathrm{ex}, 1}=2 \pi \mathrm{GHz}, \kappa_{\mathrm{ex}, 2}=2 \pi \times 5 \mathrm{GHz}, \kappa_{i}=2 \pi \times 100 \mathrm{MHz}, g_{1}(0)=\kappa_{\mathrm{ex}, 1}=g_{2}(0), \alpha_{1}(0)=0.6535, \alpha_{2}(0)=-0.6560$, and $\beta_{\alpha}(0)=0.2970$. 


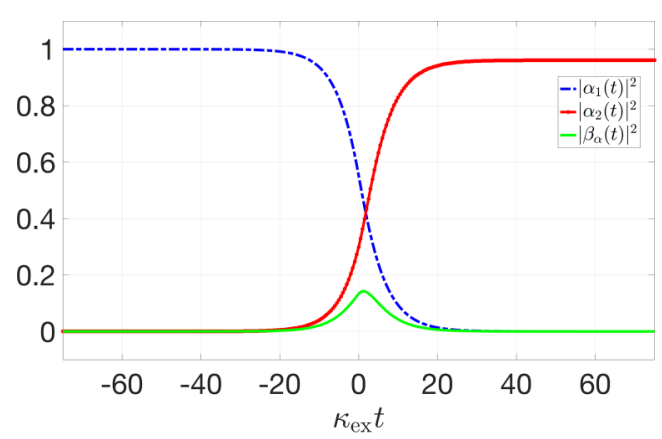

(a)

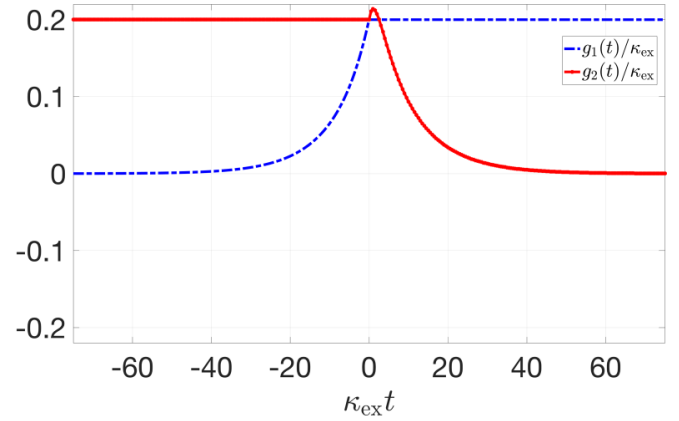

(b)

FIG. 6. Evolution of the state populations $\left|\alpha_{1}(t)\right|^{2},\left|\alpha_{2}(t)\right|^{2}$, and $\left|\beta_{\alpha}(t)\right|^{2}$ (a) and optimal pulse profiles $g_{1}(t)$ and $g_{2}(t)$ (b) over time for $\kappa_{\mathrm{ex}}=\kappa_{\mathrm{ex}, 1}=\kappa_{\mathrm{ex}, 2}=2 \pi \times 5 \mathrm{GHz}, \kappa_{i}=2 \pi \times 100 \mathrm{MHz}, g_{1}(0)=0.2 \kappa_{\mathrm{ex}}=g_{2}(0), \alpha_{1}(0)=0.7435, \alpha_{2}(0)=0.5470$, and $\beta_{\alpha}(0)=-0.3650$.

Lastly, we make a brief note about the meaning of the time arguments for the coupling profiles and state coefficient functions. As initially discussed in Sec. II, since the two blocks are spatially separated, the intermediate state takes some time $\tau$ to travel from $\mathrm{S} 1$ to $\mathrm{S} 2$, and therefore the clock for the $S 2$ functions will lag that for the $S 1$ functions by $\tau$.

\section{DISCUSSION AND CONCLUSIONS}

We derived the analytical expressions for the coupling coefficient control sequences that accomplish the optimal quantum state transfer fidelity for the most general model and parameters of two disparate memory blocks. The optimality is verified numerically through comprehensive simulations, resulting in $96 \%$ quantum fidelity of state transfer with practical system parameters for optomechanical memory blocks. We also studied the effect of nonidealities: intrinsic losses and asymmetric parameters of the two blocks. The fidelity exhibits degradation over the intrinsic losses, yielding a tendency to exponential decay. The obtained analytical expression for the coupling controls is widely applicable in any practical integrated quantum memory blocks with extremely long decoherence times and tunable coupling rates. We are currently in the process of building an integrated system for further experimental verification.

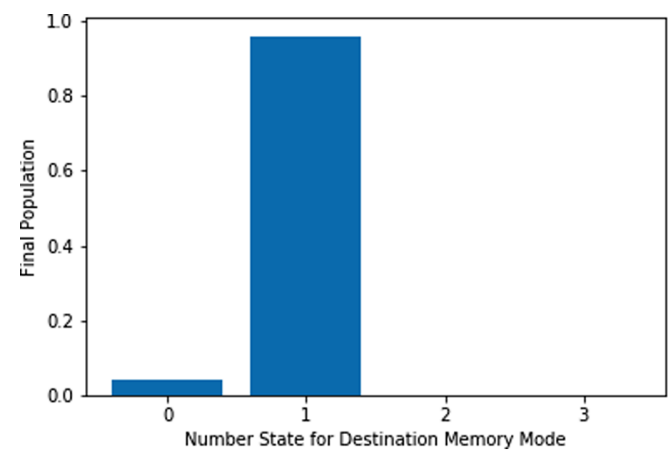

(a)
It is noteworthy that our theory is applicable to an addressable quantum memory stack with multiple memory blocks. For example, the quantum information of the S1 memory block can be transferred to a desired address of quantum memory through the coherent switching of the output $a_{\text {out, } 1}$ to an input $a_{\mathrm{in}, n}$ of the $\mathrm{S} n$ memory block via programmable nanophotonic processors [29,30]. Memory blocks may have different energy gaps between the two qubit basis states if the coupling between $a_{n}$ and $b_{n}$ fields is achieved through a parametric process where the mediating classical signal embedded in $g_{n}(t)$ may have the oscillation frequency to cancel the frequency difference between $a_{n}$ and $b_{n}$ fields.

Recent progress in noise reduction has made quantum state transfer more feasible for technological applications [31,32]. Furthermore, increasingly sophisticated types of quantum state transfer, such as frequency-tuning-induced state transfer [33] and Floquet-engineered state transfer [34], have been recently reported. More broadly, it has even been theorized recently that quantum state transfer serves as the mechanism for photosynthesis [35], thus opening up the potential for the nexus of quantum mechanics and biology. Perhaps the most immediate application is in the construction of the quantum network [36-39]. In particular, an important topic of future research is to examine the delay and backlog associated with such a network [40].

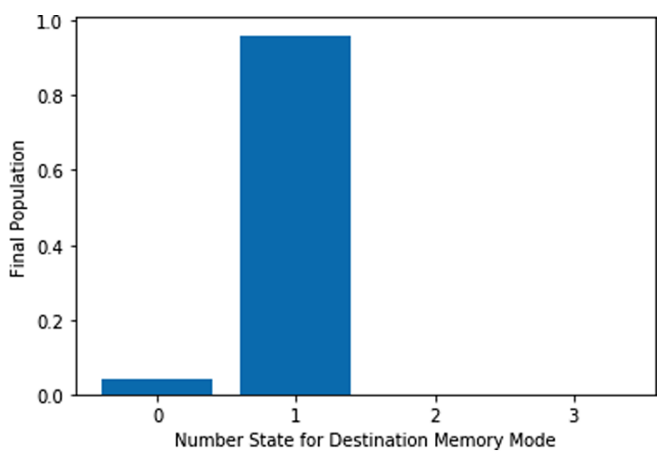

(b)

FIG. 7. Final occupation probabilities for $b_{2}$ number states for $\kappa_{\mathrm{ex}}=\kappa_{\mathrm{ex}, 1}=\kappa_{\mathrm{ex}, 2}=2 \pi \times 5 \mathrm{GHz}, \kappa_{i}=2 \pi \times 100 \mathrm{MHz}, g_{1}(0)=\kappa_{\mathrm{ex}}=$ $g_{2}(0), \alpha_{1}(0)=0.7, \alpha_{2}(0)=-0.7$, and $\beta_{\alpha}(0)=0$ (a) and $\kappa_{\mathrm{ex}}=\kappa_{\mathrm{ex}, 1}=\kappa_{\mathrm{ex}, 2}=2 \pi \times 5 \mathrm{GHz}, \kappa_{i}=2 \pi \times 100 \mathrm{MHz}, g_{1}(0)=0.2 \kappa_{\mathrm{ex}}=g_{2}(0)$, $\alpha_{1}(0)=0.7435, \alpha_{2}(0)=0.5470$, and $\beta_{\alpha}(0)=-0.3650(\mathrm{~b})$. 


\section{ACKNOWLEDGMENTS}

This work was supported by the U.S. Department of Energy, Office of Science, Advanced Scientific Computing Research (ASCR) under Award No. FWP 19-022266, "Quantum Transduction and Buffering Between Microwave Quantum Information Systems and Flying Optical Photons in Fibers." The work was also supported by the Laboratory Directed Research and Development program at Sandia National Laboratories, a multimission laboratory managed and operated by National Technology and Engineering Solutions of Sandia, LLC, a wholly owned subsidiary of Honeywell International, Inc., for the U.S. Department of Energy's National Nuclear Security Administration under Contract No. U.S. Department of Energy's National Nuclear Security Administration. This paper describes objective technical results and analysis. Any subjective views or opinions that might be expressed in the paper do not necessarily represent the views of the U.S. Department of Energy or the United States Government. M.E. performed this work, in part, at the Center for Integrated Nanotechnologies, an Office of Science User Facility operated for the U.S. Department of Energy (DOE) Office of Science.

\section{APPENDIX A: DERIVING THE COEFFICIENT EQUATIONS}

We start with the general case of asymmetric blocks with unrelated rates of intermediate state extrinsic loss (also referred to as the output coupling rate elsewhere), i.e., $\kappa_{\mathrm{ex}}^{\prime}$ does not necessarily equal $\kappa_{\mathrm{ex}}$. For the purpose of this analysis, we will define the dimensionless constant $\epsilon=\kappa_{\mathrm{ex}}^{\prime} / \kappa_{\mathrm{ex}}$, which would reduce to 1 if the two blocks were identical. As discussed in the main text, we wish to keep the system in a dark superposition of the intermediate states $|10\rangle$ and $|01\rangle$ at all times, such that the wave packet is never reflected from the second block. The following Lindbladian represents the loss due to wave-packet reflection:

$$
\begin{aligned}
L_{\mathrm{ex}} & =\sqrt{\kappa_{\mathrm{ex}}} a_{1}+\sqrt{\kappa_{\mathrm{ex}}^{\prime}} a_{2} \\
& =\sqrt{\kappa_{\mathrm{ex}}}\left(a_{1}+\sqrt{\epsilon} a_{2}\right) .
\end{aligned}
$$

The goal is for this Lindbladian to return a value of 0 when applied to the intermediate state part of the system wave function. To solve for this dark mode, we construct the following generic superposition of $a_{1}$ and $a_{2}$ modes:

$$
\mid \text { dark }\rangle=c_{1}|10\rangle+c_{2}|01\rangle \text {. }
$$

Applying the Lindbladian to this state and setting the output to 0 , we derive the following equation relating $c_{1}$ and $c_{2}$ :

$$
c_{1}+\sqrt{\epsilon} c_{2}=0 \text {. }
$$

The coefficients $c_{1}$ and $c_{2}$ must also satisfy the normalization condition:

$$
\left|c_{1}\right|^{2}+\left|c_{2}\right|^{2}=1
$$

Solving the above two equations and substituting into Eq. (A2), we find the dark mode wave function in terms of the two cavity modes:

$$
\mid \text { dark }\rangle=\frac{1}{\sqrt{1+\epsilon}}(|01\rangle-\sqrt{\epsilon}|10\rangle) .
$$

In order to complete the photonic Hilbert subspace, it is also necessary to define the bright mode as orthogonal to the dark mode:

$$
\mid \text { bright }\rangle=\frac{1}{\sqrt{1+\epsilon}}(\sqrt{\epsilon}|01\rangle+|10\rangle) .
$$

Next, we construct ladder operators corresponding to the new basis for the intermediate state Hilbert subspace, so that we can then rewrite the Hamiltonian in terms of those operators. We denote the annihilation operator for a dark mode as follows:

$$
a_{d}=|00\rangle\langle\text { dark }| .
$$

Similarly, the annihilation operator for a bright mode is defined in the following manner:

$$
a_{b}=|00\rangle\langle\text { bright }| \text {. }
$$

From the dark and bright mode wave functions, we know that these ladder operators relate to $a_{1}$ and $a_{2}$ through the following system of equations:

$$
\begin{aligned}
& a_{d}=\frac{1}{\sqrt{1+\epsilon}}\left(a_{2}-\sqrt{\epsilon} a_{1}\right), \\
& a_{b}=\frac{1}{\sqrt{1+\epsilon}}\left(\sqrt{\epsilon} a_{2}+a_{1}\right) .
\end{aligned}
$$

We solve this system to find $a_{1}$ and $a_{2}$ in terms of $a_{b}$ and $a_{d}$ :

$$
\begin{aligned}
& a_{1}=\frac{1}{\sqrt{1+\epsilon}}\left(a_{b}-\sqrt{\epsilon} a_{d}\right), \\
& a_{2}=\frac{1}{\sqrt{1+\epsilon}}\left(\sqrt{\epsilon} a_{b}+a_{d}\right) .
\end{aligned}
$$

We are now in a position to substitute these into the Hamiltonian. Assuming equal rates of intrinsic loss (since we construct both blocks to minimize this rate) but unrelated rates of extrinsic loss, the Hamiltonian takes the following form in terms of $a_{1}$ and $a_{2}$ :

$$
\begin{aligned}
H_{T}^{\mathrm{eff}}= & -\hbar g_{1}(t)\left(a_{1}^{\dagger} b_{1}+a_{1} b_{1}^{\dagger}\right)-\hbar g_{2}(t)\left(a_{2}^{\dagger} b_{2}+a_{2} b_{2}^{\dagger}\right) \\
& +\frac{i \hbar}{2} \sqrt{\kappa_{\mathrm{ex}} \kappa_{\mathrm{ex}}^{\prime}}\left(a_{1}^{\dagger} a_{2}-a_{1} a_{2}^{\dagger}\right) \\
& -\frac{i \hbar}{2}\left(\sqrt{\kappa_{\mathrm{ex}}} a_{1}^{\dagger}+\sqrt{\kappa_{\mathrm{ex}}^{\prime}} a_{2}^{\dagger}\right)\left(\sqrt{\kappa_{\mathrm{ex}}} a_{1}+\sqrt{\kappa_{\mathrm{ex}}^{\prime}} a_{2}\right) \\
& -i \hbar \frac{\kappa_{i}}{2}\left(a_{1}^{\dagger} a_{1}+a_{2}^{\dagger} a_{2}\right)-i \hbar \frac{\Gamma_{i}}{2}\left(b_{1}^{\dagger} b_{1}+b_{2}^{\dagger} b_{2}\right) .
\end{aligned}
$$

Substituting Eq. (A11) and (A12) term by term,

$$
a_{1}^{\dagger} b_{1}+a_{1} b_{1}^{\dagger}=\frac{1}{\sqrt{1+\epsilon}}\left(a_{b}^{\dagger} b_{1}-\sqrt{\epsilon} a_{d}^{\dagger} b_{1}+a_{b} b_{1}^{\dagger}-\sqrt{\epsilon} a_{d} b_{1}^{\dagger}\right),
$$

$$
a_{2}^{\dagger} b_{2}+a_{2} b_{2}^{\dagger}=\frac{1}{\sqrt{1+\epsilon}}\left(\sqrt{\epsilon} a_{b}^{\dagger} b_{2}+a_{d}^{\dagger} b_{2}+\sqrt{\epsilon} a_{b} b_{2}^{\dagger}+a_{d} b_{2}^{\dagger}\right),
$$




$$
\begin{aligned}
a_{1}^{\dagger} a_{2}-a_{1} a_{2}^{\dagger} & =\frac{1}{1+\epsilon}\left(a_{b}^{\dagger} a_{d}-a_{b} a_{d}^{\dagger}-\epsilon a_{b} a_{d}^{\dagger}+\epsilon a_{b}^{\dagger} a_{d}\right) \\
& =\frac{1}{1+\epsilon}(1+\epsilon)\left(a_{b}^{\dagger} a_{d}-a_{b} a_{d}^{\dagger}\right) \\
& =a_{b}^{\dagger} a_{d}-a_{b} a_{d}^{\dagger} .
\end{aligned}
$$

From Eqs. (A9) and (A10),

$$
\left(a_{1}^{\dagger}+\sqrt{\epsilon} a_{2}^{\dagger}\right)\left(a_{1}+\sqrt{\epsilon} a_{2}\right)=(1+\epsilon) a_{b}^{\dagger} a_{b} .
$$

Since the total intermediate state occupation number for bright and dark modes is the same as the total for the two cavity modes,

$$
a_{1}^{\dagger} a_{1}+a_{2}^{\dagger} a_{2}=a_{b}^{\dagger} a_{b}+a_{d}^{\dagger} a_{d} .
$$

These yield the following expression for the Hamiltonian in terms of the bright and dark state ladder operators:

$$
\begin{aligned}
H_{T}^{\mathrm{eff}}= & \hbar g_{1}(t) \frac{\sqrt{\epsilon}}{\sqrt{1+\epsilon}}\left(a_{d}^{\dagger} b_{1}+a_{d} b_{1}^{\dagger}\right)-\hbar g_{1}(t) \frac{1}{\sqrt{1+\epsilon}}\left(a_{b}^{\dagger} b_{1}+a_{b} b_{1}^{\dagger}\right)-\hbar g_{2}(t) \frac{1}{\sqrt{1+\epsilon}}\left(a_{d}^{\dagger} b_{2}+a_{d} b_{2}^{\dagger}\right)-\hbar g_{2}(t) \\
& \times \frac{\sqrt{\epsilon}}{\sqrt{1+\epsilon}}\left(a_{b}^{\dagger} b_{2}+a_{b} b_{2}^{\dagger}\right)+i \hbar \frac{\kappa_{\mathrm{ex}}}{2} \sqrt{\epsilon}\left(a_{b}^{\dagger} a_{d}-a_{b} a_{d}^{\dagger}\right)-i \hbar \frac{\kappa_{\mathrm{ex}}}{2}(1+\epsilon) a_{b}^{\dagger} a_{b}-i \hbar \frac{\kappa_{i}}{2}\left(a_{b}^{\dagger} a_{b}+a_{d}^{\dagger} a_{d}\right)-i \hbar \frac{\Gamma_{i}}{2}\left(b_{1}^{\dagger} b_{1}+b_{2}^{\dagger} b_{2}\right)
\end{aligned}
$$

Since the system is always in a dark state, it is useful to consider only the matrix elements of the Hamiltonian corresponding to a dark initial state and thereby omit all terms that annihilate the bright mode or return the bright mode occupation number:

$$
\begin{aligned}
H_{T}^{\mathrm{eff}}|\Psi(t)\rangle\langle\Psi(t)|= & \hbar g_{1}(t) \frac{\sqrt{\epsilon}}{\sqrt{1+\epsilon}}\left(a_{d}^{\dagger} b_{1}+a_{d} b_{1}^{\dagger}\right)-\hbar g_{1}(t) \frac{1}{\sqrt{1+\epsilon}} a_{b}^{\dagger} b_{1}-\hbar g_{2}(t) \frac{1}{\sqrt{1+\epsilon}}\left(a_{d}^{\dagger} b_{2}+a_{d} b_{2}^{\dagger}\right)-\hbar g_{2}(t) \frac{\sqrt{\epsilon}}{\sqrt{1+\epsilon}} a_{b}^{\dagger} b_{2} \\
& +i \hbar \frac{\kappa_{\mathrm{ex}}}{2} \sqrt{\epsilon} a_{b}^{\dagger} a_{d}-i \hbar \frac{\kappa_{i}}{2} a_{d}^{\dagger} a_{d}-i \hbar \frac{\Gamma_{i}}{2}\left(b_{1}^{\dagger} b_{1}+b_{2}^{\dagger} b_{2}\right) .
\end{aligned}
$$

Importantly, the non-Hermitian term corresponding to loss due to wave-packet reflection, i.e., $-i \hbar \frac{\kappa_{\mathrm{ex}}}{2}(1+\epsilon) a_{b}^{\dagger} a_{b}$, goes to 0 for the dark mode, thus validating our original method of rotating the basis for the photonic Hilbert subspace.

Next, we use the Schrödinger equation to establish a system of equations for the time-evolving state coefficients $\alpha_{1}(t)$, $\alpha_{2}(t)$, and $\beta_{\alpha}(t)$. Recall that the system wave function at any time $t$ can be expressed as follows:

$$
\begin{aligned}
|\Psi(t)\rangle= & c_{g}|g g\rangle|00\rangle+c_{e}\left(\alpha_{1}(t)|e g\rangle|00\rangle\right. \\
& \left.\left.+\alpha_{2}(t)|g e\rangle|00\rangle+i \beta_{\alpha}(t)|g g\rangle \mid \text { dark }\right\rangle\right) .
\end{aligned}
$$

Solving the matrix Schrödinger equation row by row, we obtain the following expressions:

$$
\left.\begin{array}{rl}
i \hbar c_{e} \dot{\alpha}_{1}(t) & =\left\langle e g, 00\left|H_{T}^{\mathrm{eff}}\right| \Psi(t)\right\rangle \\
& =c_{e}\left(\hbar g_{1}(t) \frac{\sqrt{\epsilon}}{\sqrt{1+\epsilon}}\left[i \beta_{\alpha}(t)\right]-i \hbar \frac{\Gamma_{i}}{2} \alpha_{1}(t)\right), \\
i \hbar c_{e} \dot{\alpha}_{2}(t) & =\left\langle g e, 00\left|H_{T}^{\mathrm{eff}}\right| \Psi(t)\right\rangle \\
& =c_{e}\left(-\hbar g_{2}(t) \frac{1}{\sqrt{1+\epsilon}}\left[i \beta_{\alpha}(t)\right]-i \hbar \frac{\Gamma_{i}}{2} \alpha_{2}(t)\right), \\
-\hbar c_{e} \beta_{\alpha}(t) & =\left\langle g g, \operatorname{dark}\left|H_{T}^{\mathrm{eff}}\right| \Psi(t)\right\rangle \\
& =c_{e}\left(\hbar g_{1}(t) \frac{\sqrt{\epsilon}}{\sqrt{1+\epsilon}} \alpha_{1}(t)-\hbar g_{2}(t) \frac{1}{\sqrt{1+\epsilon}} \alpha_{2}(t)\right.
\end{array}\right) .
$$

It is also necessary for the coupling profiles to satisfy the following equation in order to prevent the Hamiltonian from coupling the system into a bright mode:

$$
\begin{aligned}
0 & =\left\langle g g, \operatorname{bright}\left|H_{T}^{\mathrm{eff}}\right| \Psi(t)\right\rangle \\
& =c_{e}\left(\begin{array}{c}
-\hbar g_{1}(t) \frac{1}{\sqrt{1+\epsilon}} \alpha_{1}(t)-\hbar g_{2}(t) \frac{\sqrt{\epsilon}}{\sqrt{1+\epsilon}} \alpha_{2}(t) \\
+i \hbar \frac{\kappa_{\mathrm{ex}}}{2} \sqrt{\epsilon}\left[i \beta_{\alpha}(t)\right]
\end{array}\right) .
\end{aligned}
$$

Dividing the first two equations by $i \hbar c_{e}$ and the latter two by $-\hbar c_{e}$, we find a system of differential equations for the time evolution of the coefficients:

$$
\begin{gathered}
\dot{\alpha}_{1}(t)=\frac{\sqrt{\epsilon}}{\sqrt{1+\epsilon}} g_{1}(t) \beta_{\alpha}(t)-\frac{\Gamma_{i}}{2} \alpha_{1}(t), \\
\dot{\alpha}_{2}(t)=-\frac{1}{\sqrt{1+\epsilon}} g_{2}(t) \beta_{\alpha}(t)-\frac{\Gamma_{i}}{2} \alpha_{2}(t), \\
\dot{\beta_{\alpha}}(t)=\frac{1}{\sqrt{1+\epsilon}} g_{2}(t) \alpha_{2}(t)-\frac{\sqrt{\epsilon}}{\sqrt{1+\epsilon}} g_{1}(t) \alpha_{1}(t)-\frac{\kappa_{i}}{2} \beta_{\alpha}(t), \\
0=\frac{\sqrt{\epsilon}}{\sqrt{1+\epsilon}} g_{2}(t) \alpha_{2}(t)+\frac{1}{\sqrt{1+\epsilon}} g_{1}(t) \alpha_{1}(t)+\frac{\kappa_{\mathrm{ex}} \sqrt{\epsilon}}{2} \beta_{\alpha}(t) .
\end{gathered}
$$

\section{APPENDIX B: SOLVING FOR THE SECOND HALF OF THE PROCESS}

We will start by solving the coefficients for $t \geqslant 0$ (i.e., the second half of the transfer process), setting $g_{1}(t)=g_{1}(0)$ for this time range. Since the system in its final state has either fully entered the $b_{2}$ phonon mode $|g e\rangle|00\rangle$ or has collapsed to the vacuum state $|g g\rangle|00\rangle, \alpha_{1}(t)$ and $\beta_{\alpha}(t)$ will converge to 0 as $t \rightarrow \infty$, and we therefore start by solving for those coefficients. The coupling profile $g_{2}(t)$ can be determined in terms of the state coefficients and the constant $g_{1}(0)$ by 
rearranging Eq. (A29) as follows:

$$
\begin{aligned}
g_{2}(t) & =-\frac{\sqrt{1+\epsilon}}{\sqrt{\epsilon}} \frac{\left(\frac{\kappa_{\mathrm{ex}} \sqrt{\epsilon}}{2} \beta_{\alpha}(t)+\frac{1}{\sqrt{1+\epsilon}} g_{1}(0) \alpha_{1}(t)\right)}{\alpha_{2}(t)} \\
& =-\frac{\left(\frac{\kappa_{\mathrm{ex}} \sqrt{1+\epsilon}}{2} \beta_{\alpha}(t)+\frac{1}{\sqrt{\epsilon}} g_{1}(0) \alpha_{1}(t)\right)}{\alpha_{2}(t)} .
\end{aligned}
$$

Substituting this into Eq. (A28), we find the following expression for $\dot{\beta}_{\alpha}(t)$ in terms of $\alpha_{1}(t)$ and $\beta_{\alpha}(t)$ :

$$
\begin{aligned}
\dot{\beta}_{\alpha}(t)= & \frac{1}{\sqrt{1+\epsilon}}\left(-\frac{\kappa_{\mathrm{ex}} \sqrt{1+\epsilon}}{2} \beta_{\alpha}(t)-\frac{1}{\sqrt{\epsilon}} g_{1}(0) \alpha_{1}(t)\right) \\
& -\frac{\sqrt{\epsilon}}{\sqrt{1+\epsilon}} g_{1}(0) \alpha_{1}(t)-\frac{\kappa_{i}}{2} \beta_{\alpha}(t) \\
= & -\frac{1}{\sqrt{1+\epsilon}}\left(\frac{1}{\sqrt{\epsilon}}+\sqrt{\epsilon}\right) g_{1}(0) \alpha_{1}(t)-\frac{\kappa_{\mathrm{ex}}+\kappa_{i}}{2} \beta_{\alpha}(t) \\
= & -\frac{\sqrt{1+\epsilon}}{\sqrt{\epsilon}} g_{1}(0) \alpha_{1}(t)-\frac{\kappa_{\mathrm{ex}}+\kappa_{i}}{2} \beta_{\alpha}(t) .
\end{aligned}
$$

Combining Eqs. (A26) and (B2), we express the resulting system of differential equations as a matrix ordinary differential equation (ODE):

$$
\left(\begin{array}{c}
\dot{\alpha_{1}(t)} \\
\dot{\beta}_{\alpha}(t)
\end{array}\right)=\left(\begin{array}{cc}
-\frac{\Gamma_{i}}{2} & \frac{\sqrt{\epsilon}}{\sqrt{1+\epsilon}} g_{1}(0) \\
-\frac{\sqrt{1+\epsilon}}{\sqrt{\epsilon}} & -\frac{\kappa_{\mathrm{ex}}+\kappa_{i}}{2}
\end{array}\right)\left(\begin{array}{l}
\alpha_{1}(t) \\
\beta_{\alpha}(t)
\end{array}\right) .
$$

The eigenvalues turn out to be independent of $\epsilon$ :

$$
\lambda_{ \pm}=-\frac{1}{4}\left(\Gamma_{i}+\kappa_{\mathrm{ex}}+\kappa_{i}\right) \pm \frac{C}{4} .
$$

Here, $C=\sqrt{\left(\Gamma_{i}-\kappa_{\mathrm{ex}}-\kappa_{i}\right)^{2}-16 g_{1}(0)^{2}}$. The corresponding eigenvectors, on the other hand, vary with $\epsilon$ in the following manner:

$$
v_{ \pm}=\left(\begin{array}{c}
\left(\Gamma_{i}-\kappa_{\mathrm{ex}}-\kappa_{i} \mp C\right) \sqrt{\epsilon} \\
4 g_{1}(0) \sqrt{1+\epsilon}
\end{array}\right) .
$$

Recall that the solutions for a matrix ODE are functions of the eigenvalues, eigenvectors, and constants that are to be determined from the boundary values:

$$
\left(\begin{array}{l}
\alpha_{1}(t) \\
\beta_{\alpha}(t)
\end{array}\right)=A e^{\lambda_{-} t} v_{-}+B e^{\lambda_{+} t} v_{+} .
$$

It is worth noting that since the eigenvalues are independent of $\epsilon$, the time dependence of the individual terms in the solutions will also be invariant in $\epsilon$. Instead, only the constant coefficient in each term will change. This is evident from expressing the solutions in the following form:

$$
\begin{aligned}
\left(\begin{array}{l}
\alpha_{1}(t) \\
\beta_{\alpha}(t)
\end{array}\right)= & e^{-\frac{1}{4}\left(\Gamma_{i}+\kappa_{\mathrm{ex}}+\kappa_{i}\right) t}\left(A e^{-\frac{C}{4} t} v_{-}+B e^{\frac{C}{4} t} v_{+}\right) \\
= & e^{-\frac{1}{4}\left(\Gamma_{i}+\kappa_{\mathrm{ex}}+\kappa_{i}\right) t}\left[\left(A v_{-}+B v_{+}\right) \cosh \left(\frac{C}{4} t\right)\right. \\
& \left.+\left(B v_{+}-A v_{-}\right) \sinh \left(\frac{C}{4} t\right)\right] .
\end{aligned}
$$

The generic matrix ODE solution leads to the following system of equations for $\alpha_{1}(0)$ and $\beta_{\alpha}(0)$ in terms of $A$ and $B$ :

$$
\alpha_{1}(0)=\left[\left(\Gamma_{i}-\kappa_{\mathrm{ex}}-\kappa_{i}+C\right) A+\left(\Gamma_{i}-\kappa_{\mathrm{ex}}-\kappa_{i}-C\right) B\right] \sqrt{\epsilon},
$$

$$
\beta_{\alpha}(0)=4 g_{1}(0) \sqrt{1+\epsilon}(A+B) .
$$

Solving this system of equations, we find the following expressions for $A$ and $B$ :

$$
\begin{aligned}
A & =\frac{1}{2 C}\left(\frac{1}{\sqrt{\epsilon}} \alpha_{1}(0)-\frac{\Gamma_{i}-\kappa_{\mathrm{ex}}-\kappa_{i}-C}{4 g_{1}(0) \sqrt{1+\epsilon}} \beta_{\alpha}(0)\right), \\
B & =\frac{1}{2 C}\left(-\frac{1}{\sqrt{\epsilon}} \alpha_{1}(0)+\frac{\Gamma_{i}-\kappa_{\mathrm{ex}}-\kappa_{i}+C}{4 g_{1}(0) \sqrt{1+\epsilon}} \beta_{\alpha}(0)\right) .
\end{aligned}
$$

We first focus on solving $\alpha_{1}(t)$ by substituting $A, B, v_{-, 1}$, and $v_{+, 1}$ into the constant-coefficient expressions in Eq. (B7):

$$
\begin{aligned}
A v_{-, 1}+B v_{+, 1}= & \frac{1}{2 C}\left(\frac{1}{\sqrt{\epsilon}} \alpha_{1}(0)-\frac{\Gamma_{i}-\kappa_{\mathrm{ex}}-\kappa_{i}-C}{4 g_{1}(0) \sqrt{1+\epsilon}} \beta_{\alpha}(0)\right)\left(\Gamma_{i}-\kappa_{\mathrm{ex}}-\kappa_{i}+C\right) \sqrt{\epsilon} \\
& +\frac{1}{2 C}\left(\frac{-1}{\sqrt{\epsilon}} \alpha_{1}(0)+\frac{\Gamma_{i}-\kappa_{\mathrm{ex}}-\kappa_{i}+C}{4 g_{1}(0) \sqrt{1+\epsilon}} \beta_{\alpha}(0)\right)\left(\Gamma_{i}-\kappa_{\mathrm{ex}}-\kappa_{i}-C\right) \sqrt{\epsilon} \\
= & \frac{1}{2 C}\left(\frac{2}{\sqrt{\epsilon}} \alpha_{1}(0) C \sqrt{\epsilon}\right) \\
= & \alpha_{1}(0), \\
B v_{+, 1}-A v_{-, 1}= & \frac{1}{2 C}\left(\frac{-1}{\sqrt{\epsilon}} \alpha_{1}(0)+\frac{\Gamma_{i}-\kappa_{\mathrm{ex}}-\kappa_{i}+C}{4 g_{1}(0) \sqrt{1+\epsilon}} \beta_{\alpha}(0)\right)\left(\Gamma_{i}-\kappa_{\mathrm{ex}}-\kappa_{i}-C\right) \sqrt{\epsilon} \\
& -\frac{1}{2 C}\left(\frac{1}{\sqrt{\epsilon}} \alpha_{1}(0)-\frac{\Gamma_{i}-\kappa_{\mathrm{ex}}-\kappa_{i}-C}{4 g_{1}(0) \sqrt{1+\epsilon}} \beta_{\alpha}(0)\right)\left(\Gamma_{i}-\kappa_{\mathrm{ex}}-\kappa_{i}+C\right) \sqrt{\epsilon} \\
= & \frac{1}{2 C}\left(-2\left(\Gamma_{i}-\kappa_{\mathrm{ex}}-\kappa_{i}\right) \alpha_{1}(0)+\frac{\left(\Gamma_{i}-\kappa_{\mathrm{ex}}-\kappa_{i}\right)^{2}-C^{2}}{2 g_{1}(0)} \frac{\sqrt{\epsilon}}{\sqrt{1+\epsilon}} \beta_{\alpha}(0)\right)
\end{aligned}
$$




$$
\begin{aligned}
& =\frac{1}{2 C}\left(2\left(\kappa_{\mathrm{ex}}+\kappa_{i}-\Gamma_{i}\right) \alpha_{1}(0)+\frac{16 g_{1}(0)^{2}}{2 g_{1}(0)} \frac{\sqrt{\epsilon}}{\sqrt{1+\epsilon}} \beta_{\alpha}(0)\right) \\
& =\frac{1}{C}\left(\left(\kappa_{\mathrm{ex}}+\kappa_{i}-\Gamma_{i}\right) \alpha_{1}(0)+4 g_{1}(0) \sqrt{\frac{\epsilon}{1+\epsilon}} \beta_{\alpha}(0)\right) .
\end{aligned}
$$

Substituting these into Eq. (B7), we find the following expression for $\alpha_{1}(t)$ for $t \geqslant 0$ :

$$
\begin{aligned}
\alpha_{1}(t) & =e^{-\frac{1}{4}\left(\Gamma_{i}+\kappa_{\mathrm{ex}}+\kappa_{i}\right) t}\left[\left(A v_{-, 1}+B v_{+, 1}\right) \cosh \left(\frac{C}{4} t\right)+\left(B v_{+, 1}-A v_{-, 1}\right) \sinh \left(\frac{C}{4} t\right)\right] \\
& =\frac{e^{-\frac{1}{4}\left(\kappa_{\mathrm{ex}}+\kappa_{i}+\Gamma_{i}\right) t}}{C}\left[\alpha_{1}(0) C \cosh \left(\frac{C}{4} t\right)+\left(4 \sqrt{\frac{\epsilon}{1+\epsilon}} g_{1}(0) \beta_{\alpha}(0)+\alpha_{1}(0)\left(\kappa_{\mathrm{ex}}+\kappa_{i}-\Gamma_{i}\right)\right) \sinh \left(\frac{C}{4} t\right)\right] .
\end{aligned}
$$

Note that only the coefficient in the second term in this expression varies with $\epsilon$. When $\epsilon=1$, that coefficient reduces to $2 \sqrt{2} g_{1}(0) \beta_{\alpha}(0)$.

We now complete an analogous set of steps to determine $\beta_{\alpha}(t)$. Substituting $A, B, v_{-, 2}$, and $v_{+, 2}$ into the constant-coefficient expressions in Eq. (B7),

$$
\begin{aligned}
A v_{-, 2}+B v_{+, 2}= & \frac{1}{2 C}\left(\frac{1}{\sqrt{\epsilon}} \alpha_{1}(0)-\frac{\Gamma_{i}-\kappa_{\mathrm{ex}}-\kappa_{i}-C}{4 g_{1}(0) \sqrt{1+\epsilon}} \beta_{\alpha}(0)\right) 4 g_{1}(0) \sqrt{1+\epsilon} \\
& +\frac{1}{2 C}\left(\frac{-1}{\sqrt{\epsilon}} \alpha_{1}(0)+\frac{\Gamma_{i}-\kappa_{\mathrm{ex}}-\kappa_{i}+C}{4 g_{1}(0) \sqrt{1+\epsilon}} \beta_{\alpha}(0)\right) 4 g_{1}(0) \sqrt{1+\epsilon} \\
= & \frac{1}{2 C}\left(\frac{2 C}{4 g_{1}(0) \sqrt{1+\epsilon}} \beta_{\alpha}(0)\right) 4 g_{1}(0) \sqrt{1+\epsilon} \\
= & \beta_{\alpha}(0), \\
B v_{+, 2}-A v_{-, 2}= & \frac{1}{2 C}\left(\frac{-1}{\sqrt{\epsilon}} \alpha_{1}(0)+\frac{\Gamma_{i}-\kappa_{\mathrm{ex}}-\kappa_{i}+C}{4 g_{1}(0) \sqrt{1+\epsilon}} \beta_{\alpha}(0)\right) 4 g_{1}(0) \sqrt{1+\epsilon} \\
& -\frac{1}{2 C}\left(\frac{1}{\sqrt{\epsilon}} \alpha_{1}(0)-\frac{\Gamma_{i}-\kappa_{\mathrm{ex}}-\kappa_{i}-C}{4 g_{1}(0) \sqrt{1+\epsilon}} \beta_{\alpha}(0)\right) 4 g_{1}(0) \sqrt{1+\epsilon} \\
= & \frac{1}{2 C}\left(\frac{-2}{\sqrt{\epsilon}} \alpha_{1}(0)+\frac{\Gamma_{i}-\kappa_{\mathrm{ex}}-\kappa_{i}}{2 g_{1}(0) \sqrt{1+\epsilon}} \beta_{\alpha}(0)\right) 4 g_{1}(0) \sqrt{1+\epsilon} \\
= & -\frac{1}{C}\left(4 \sqrt{\frac{1+\epsilon}{\epsilon}} g_{1}(0) \alpha_{1}(0)+\beta_{\alpha}(0)\left(\kappa_{\mathrm{ex}}+\kappa_{i}-\Gamma_{i}\right)\right) .
\end{aligned}
$$

We substitute these into Eq. (B7) to find $\beta_{\alpha}(t)$ for $t \geqslant 0$ as follows:

$$
\begin{aligned}
\beta_{\alpha}(t) & =e^{-\frac{1}{4}\left(\Gamma_{i}+\kappa_{\mathrm{ex}}+\kappa_{i}\right) t}\left[\left(A v_{-, 2}+B v_{+, 2}\right) \cosh \left(\frac{C}{4} t\right)+\left(B v_{+, 2}-A v_{-, 2}\right) \sinh \left(\frac{C}{4} t\right)\right] \\
& =\frac{e^{-\frac{1}{4}\left(\kappa_{\mathrm{ex}}+\kappa_{i}+\Gamma_{i}\right) t}}{C}\left[\beta_{\alpha}(0) C \cosh \left(\frac{C}{4} t\right)-\left(4 \sqrt{\frac{1+\epsilon}{\epsilon}} g_{1}(0) \alpha_{1}(0)+\beta_{\alpha}(0)\left(\kappa_{\mathrm{ex}}+\kappa_{i}-\Gamma_{i}\right)\right) \sinh \left(\frac{C}{4} t\right)\right] .
\end{aligned}
$$

Note that only the second term (i.e., the first term in the square brackets) varies with $\epsilon$. When $\epsilon=1$, the coefficient in that term reduces to $4 \sqrt{2} g_{1}(0) \alpha_{1}(0)$.

We finally undertake the process of solving for $\alpha_{2}(t)$. Substituting the expression for $g_{2}(t)$ from Eq. (B1) into that for $\dot{\alpha}_{2}(t)$ from Eq. (A27), we find the following differential equation:

$$
\begin{aligned}
\dot{\alpha}_{2}(t) & =-\frac{1}{\sqrt{1+\epsilon}} \frac{\left(-\frac{\kappa_{\mathrm{ex}} \sqrt{1+\epsilon}}{2} \beta_{\alpha}(t)-\frac{1}{\sqrt{\epsilon}} g_{1}(0) \alpha_{1}(t)\right)}{\alpha_{2}(t)} \beta_{\alpha}(t)-\frac{\Gamma_{i}}{2} \alpha_{2}(t) \\
& =\frac{\left(\frac{\kappa_{\mathrm{ex}}}{2} \beta_{\alpha}(t)+\frac{g_{1}(0)}{\sqrt{\epsilon(1+\epsilon)}} \alpha_{1}(t)\right)}{\alpha_{2}(t)} \beta_{\alpha}(t)-\frac{\Gamma_{i}}{2} \alpha_{2}(t) .
\end{aligned}
$$

Multiplying both sides by $2 \alpha_{2}(t)$, the expression becomes a first-order ordinary differential equation in $\alpha_{2}^{2}$ :

$$
\frac{d}{d t} \alpha_{2}(t)^{2}=2 \alpha_{2}(t) \dot{\alpha}_{2}(t)=\left(\kappa_{\mathrm{ex}} \beta_{\alpha}(t)^{2}+\frac{2 g_{1}(0)}{\sqrt{\epsilon(1+\epsilon)}} \alpha_{1}(t) \beta_{\alpha}(t)\right)-\Gamma_{i} \alpha_{2}^{2}(t) .
$$


Note that the time-varying part of each of the constituent terms is invariant in $\epsilon$. We integrate the equation term by term:

$$
\alpha_{2}(t)^{2}=e^{-\Gamma_{i} t}\left(\begin{array}{l}
\kappa_{\mathrm{ex}} \int e^{\Gamma_{i} t} \beta_{\alpha}(t)^{2} d t \\
+\frac{2 g_{1}(0)}{\sqrt{\epsilon(1+\epsilon)}} \int e^{\Gamma_{i} t} \alpha_{1}(t) \beta_{\alpha}(t) d t+G
\end{array}\right) .
$$

Here, $G$ is a constant that will eventually be calculated from the boundary conditions for $\alpha_{2}(t)^{2}$. For now, we focus on the first term inside the parentheses. Our first goal is to examine $\int e^{\Gamma_{i} t} \beta_{\alpha}(t)^{2} d t$. The integrand can be expanded by substituting the expression for $\beta_{\alpha}(t)$ from Eq. (B17):

$$
e^{\Gamma_{i} t} \beta_{\alpha}(t)^{2}=\frac{e^{-\frac{1}{2}\left(\kappa_{\mathrm{ex}}+\kappa_{i}-\Gamma_{i}\right) t}}{C^{2}}\left[\beta_{\alpha}(0)^{2} C^{2} \cosh ^{2}\left(\frac{C}{4} t\right)-2 B_{1}^{\prime} \beta_{\alpha}(0) C \cosh \left(\frac{C}{4} t\right) \sinh \left(\frac{C}{4} t\right)+B_{1}^{\prime 2} \sinh ^{2}\left(\frac{C}{4} t\right)\right] .
$$

For each of the terms in Eq. (B21), we integrate the time-dependent part using MATHEMATICA and then multiply by the associated constant. For the first term, we find the following result:

$$
\begin{aligned}
\beta_{\alpha}(0)^{2} \int e^{-\frac{1}{2}\left(\kappa_{\mathrm{ex}}+\kappa_{i}-\Gamma_{i}\right) t} \cosh ^{2}\left(\frac{C}{4} t\right)= & \frac{\beta_{\alpha}(0)^{2} e^{\frac{1}{2}\left(\Gamma_{i}-\kappa_{\mathrm{ex}}-\kappa_{i}\right) t}}{\left(\Gamma_{i}-\kappa_{\mathrm{ex}}-\kappa_{i}\right)\left[\left(\Gamma_{i}-\kappa_{\mathrm{ex}}-\kappa_{i}\right)^{2}-C^{2}\right]}\left[-C^{2}-C\left(\Gamma_{i}-\kappa_{\mathrm{ex}}-\kappa_{i}\right) \sinh \left(\frac{C}{2} t\right)\right. \\
& \left.+\left(\Gamma_{i}-\kappa_{\mathrm{ex}}-\kappa_{i}\right)^{2} \cosh ^{2}\left(\frac{C}{2} t\right)+\left(\Gamma_{i}-\kappa_{\mathrm{ex}}-\kappa_{i}\right)^{2}\right]
\end{aligned}
$$

For the second term, we find the following:

$$
\begin{aligned}
- & \frac{2 B_{1}^{\prime} \beta_{\alpha}(0)}{C} \int e^{-\frac{1}{2}\left(\kappa_{\mathrm{ex}}+\kappa_{i}-\Gamma_{i}\right) t} \cosh \left(\frac{C}{4} t\right) \sinh \left(\frac{C}{4} t\right) \\
= & \frac{2 B_{1}^{\prime} \beta_{\alpha}(0) e^{\frac{1}{2}\left(\Gamma_{i}-\kappa_{\mathrm{ex}}-\kappa_{i}\right) t}}{C\left[\left(\Gamma_{i}-\kappa_{\mathrm{ex}}-\kappa_{i}\right)^{2}-C^{2}\right]}\left[\left(\kappa_{\mathrm{ex}}+\kappa_{i}-\Gamma_{i}\right) \sinh \left(\frac{C}{2} t\right)+C \cosh \left(\frac{C}{2} t\right)\right] .
\end{aligned}
$$

Finally, the third term yields the following:

$$
\begin{aligned}
\frac{B_{1}^{\prime 2}}{C^{2}} \int e^{-\frac{1}{2}\left(\kappa_{\mathrm{ex}}+\kappa_{i}-\Gamma_{i}\right) t} \sinh ^{2}\left(\frac{C}{4} t\right)= & \frac{B_{1}^{\prime 2} e^{\frac{1}{2}\left(\Gamma_{i}-\kappa_{\mathrm{ex}}-\kappa_{i}\right) t}}{C^{2}\left(\Gamma_{i}-\kappa_{\mathrm{ex}}-\kappa_{i}\right)\left[\left(\Gamma_{i}-\kappa_{\mathrm{ex}}-\kappa_{i}\right)^{2}-C^{2}\right]}\left[C^{2}-C\left(\Gamma_{i}-\kappa_{\mathrm{ex}}-\kappa_{i}\right) \sinh \left(\frac{C}{2} t\right)\right. \\
& \left.+\left(\Gamma_{i}-\kappa_{\mathrm{ex}}-\kappa_{i}\right)^{2} \cosh ^{2}\left(\frac{C}{2} t\right)-\left(\Gamma_{i}-\kappa_{\mathrm{ex}}-\kappa_{i}\right)^{2}\right]
\end{aligned}
$$

Note that all three of these expressions contain the term $\left(\Gamma_{i}-\kappa_{\mathrm{ex}}-\kappa_{i}\right)^{2}-C^{2}$ in the denominator, which simply equals $16 g_{1}(0)^{2}$. Summing the three expressions, we find the following result for $\int e^{\Gamma_{i} t} \beta_{\alpha}(t)^{2} d t$ :

$$
\int e^{\Gamma_{i} t} \beta_{\alpha}(t)^{2} d t=\frac{e^{\frac{1}{2}\left(\Gamma_{i}-\kappa_{\mathrm{ex}}-\kappa_{i}\right) t}}{16 g_{1}(0)^{2}}\left[\beta_{\alpha}(0)^{2} A_{1}(t)+2 B_{1}^{\prime} \beta_{\alpha}(0) A_{2}(t)+B_{1}^{\prime 2} A_{3}(t)\right],
$$

where we have defined $A_{1}(t), A_{2}(t)$, and $A_{3}(t)$ as

$$
\begin{gathered}
A_{1}(t)=\frac{-C^{2}-C\left(\Gamma_{i}-\kappa_{i}-\kappa_{\mathrm{ex}}\right) \sinh \left(\frac{C}{2} t\right)+\left(\Gamma_{i}-\kappa_{i}-\kappa_{\mathrm{ex}}\right)^{2}\left[\cosh \left(\frac{C}{2} t\right)+1\right]}{\Gamma_{i}-\kappa_{i}-\kappa_{\mathrm{ex}}}, \\
A_{2}(t)=\frac{-\left(\Gamma_{i}-\kappa_{i}-\kappa_{\mathrm{ex}}\right) \sinh \left(\frac{C}{2} t\right)+C \cosh \left(\frac{C}{2} t\right)}{C} \\
A_{3}(t)=\frac{C^{2}-C\left(\Gamma_{i}-\kappa_{i}-\kappa_{\mathrm{ex}}\right) \sinh \left(\frac{C}{2} t\right)+\left(\Gamma_{i}-\kappa_{i}-\kappa_{\mathrm{ex}}\right)^{2}\left[\cosh \left(\frac{C}{2} t\right)-1\right]}{C^{2}\left(\Gamma_{i}-\kappa_{i}-\kappa_{\mathrm{ex}}\right)} .
\end{gathered}
$$

Next, we examine $\int e^{\Gamma_{i} t} \alpha_{1}(t) \beta_{\alpha}(t) d t$. We expand the integrand in a manner analogous to Eq. (B21), this time by substituting $\alpha_{1}(t)$ from Eq. (B14) and $\beta_{\alpha}(t)$ from Eq. (B17):

$$
\begin{aligned}
e^{\Gamma_{i} t} \alpha_{1}(t) \beta_{\alpha}(t)= & \frac{e^{-\frac{1}{2}\left(\kappa_{\mathrm{ex}}+\kappa_{i}-\Gamma_{i}\right) t}}{C^{2}}\left\{\alpha_{1}(0) \beta_{\alpha}(0) C^{2} \cosh ^{2}\left(\frac{C}{4} t\right)-\left[\alpha_{1}(0) B_{1}^{\prime}-\beta_{\alpha}(0) B_{2}^{\prime}\right]\right. \\
& \left.\times C \cosh \left(\frac{C}{4} t\right) \sinh \left(\frac{C}{4} t\right)+\left(-B_{1}^{\prime} B_{2}^{\prime}\right) \sinh ^{2}\left(\frac{C}{4} t\right)\right\} .
\end{aligned}
$$


Analyzing Eq. (B29), we observe that the form of the expression is equivalent to that for $e^{\Gamma_{i} t} \beta_{\alpha}(t)^{2}$ in Eq. (B21), with the replacements $\beta_{\alpha}(0)^{2} \rightarrow \alpha_{1}(0) \beta_{\alpha}(0)$ in the first term, $2 B_{1}^{\prime} \beta_{\alpha}(0) \rightarrow \alpha_{1}(0) B_{1}^{\prime}-\beta_{\alpha}(0) B_{2}^{\prime}$ in the second term, and $B_{1}^{\prime 2} \rightarrow-B_{1}^{\prime} B_{2}^{\prime}$ in the third term. We therefore determine $\int e^{\Gamma_{i} t} \alpha_{1}(t) \beta_{\alpha}(t) d t$ simply by using Eq. (B25) and implementing these replacements:

$$
\int e^{\Gamma_{i} t} \alpha_{1}(t) \beta_{\alpha}(t) d t=\frac{e^{\frac{1}{2}\left(\Gamma_{i}-\kappa_{\mathrm{ex}}-\kappa_{i}\right) t}}{16 g_{1}(0)^{2}}\left\{\alpha_{1}(0) \beta_{\alpha}(0) A_{1}(t)+\left[\alpha_{1}(0) B_{1}^{\prime}-\beta_{\alpha}(0) B_{2}^{\prime}\right] A_{2}(t)-B_{1}^{\prime} B_{2}^{\prime} A_{3}(t)\right\} .
$$

Superposing these integrals using the coefficients in Eq. (B20), we find the following result for $\alpha_{2}(t)^{2}$ for $t \geqslant 0$ :

$$
\begin{aligned}
\alpha_{2}(t)^{2}= & e^{-\Gamma_{i} t}\left(\kappa_{\mathrm{ex}} \int e^{\Gamma_{i} t} \beta_{\alpha}(t)^{2} d t+\frac{2 g_{1}(0)}{\sqrt{\epsilon(1+\epsilon)}} \int e^{\Gamma_{i} t} \alpha_{1}(t) \beta_{\alpha}(t) d t+G\right) \\
= & \frac{e^{-\frac{1}{2}\left(\kappa_{\mathrm{ex}}+\kappa_{i}+\Gamma_{i}\right) t}}{16 g_{1}(0)^{2}}\left\{\left(\kappa_{\mathrm{ex}} \beta_{\alpha}(0)^{2}+\frac{2 g_{1}(0)}{\sqrt{\epsilon(1+\epsilon)}} \alpha_{1}(0) \beta_{\alpha}(0)\right) A_{1}(t)\right. \\
& \left.+\left(2 \kappa_{\mathrm{ex}} B_{1}^{\prime} \beta_{\alpha}(0)+\frac{2 g_{1}(0)}{\sqrt{\epsilon(1+\epsilon)}}\left[\alpha_{1}(0) B_{1}^{\prime}-\beta_{\alpha}(0) B_{2}^{\prime}\right]\right) A_{2}(t)+\left(\kappa_{\mathrm{ex}} B_{1}^{\prime 2}-\frac{2 g_{1}(0)}{\sqrt{\epsilon(1+\epsilon)}} B_{1}^{\prime} B_{2}^{\prime}\right) A_{3}(t)\right\}+G e^{-\Gamma_{i} t} .
\end{aligned}
$$

Regarding the term $G e^{-\Gamma_{i} t}$, it is useful to note that $\Gamma_{i} \ll$ $\kappa_{\mathrm{ex}}, \kappa_{\mathrm{ex}}^{\prime}, \kappa_{i}$. Therefore, given the timescale of the transfer process, $e^{-\Gamma_{i} t}$ will approximately equal 1 . Since the first term in the $\alpha_{2}(t)^{2}$ expression converges to 0 as $t \rightarrow t_{f}, G$ will approximately equal $\alpha_{2}^{2}\left(t_{f}\right)$, representing the fidelity of states for the process.

\section{APPENDIX C: SOLVING FOR THE FIRST HALF OF THE PROCESS}

We now analyze the time range $t<0$, representing the first half of the process. Here, $g_{2}(t)=g_{2}(0)$ will be constant in time, whereas $g_{1}(t)$ will be variable. Since the system is fully in the $b_{1}$ mode initially, we know that $\alpha_{2}(t)$ and $\beta_{\alpha}(t)$ will converge to 0 for the maximally negative value of $t$. As such, we start by solving for the time evolution of those coefficients. Rearranging Eq. (A29) yields the following expression for $g_{1}(t)$ in terms of $g_{2}(0)$ and the state coefficients:

$$
\begin{aligned}
g_{1}(t) & =-\sqrt{1+\epsilon} \frac{\left(\frac{\kappa_{\mathrm{ex}} \sqrt{\epsilon}}{2} \beta_{\alpha}(t)+\frac{\sqrt{\epsilon}}{\sqrt{1+\epsilon}} g_{2}(0) \alpha_{2}(t)\right)}{\alpha_{1}(t)} \\
& =-\frac{\left(\frac{\kappa_{\mathrm{ex}} \sqrt{\epsilon(1+\epsilon)}}{2} \beta_{\alpha}(t)+\sqrt{\epsilon} g_{2}(0) \alpha_{2}(t)\right)}{\alpha_{1}(t)} .
\end{aligned}
$$

Substituting this into Eq. (A28), we find a modified differential equation for $\dot{\beta_{\alpha}}(t)$ in terms of $\alpha_{2}(t)$ and $\beta_{\alpha}(t)$ :

$$
\begin{aligned}
\dot{\beta}_{\alpha}(t) & =\frac{1}{\sqrt{1+\epsilon}} g_{2}(0) \alpha_{2}(t)-\frac{\sqrt{\epsilon}}{\sqrt{1+\epsilon}}\left(-\frac{\kappa_{\mathrm{ex}} \sqrt{\epsilon(1+\epsilon)}}{2} \beta_{\alpha}(t)-\sqrt{\epsilon} g_{2}(0) \alpha_{2}(t)\right)-\frac{\kappa_{i}}{2} \beta_{\alpha}(t) \\
& =\frac{1}{\sqrt{1+\epsilon}}(1+\epsilon) g_{2}(0) \alpha_{2}(t)-\frac{\kappa_{i}-\epsilon \kappa_{\mathrm{ex}}}{2} \beta_{\alpha}(t) \\
& =\sqrt{1+\epsilon} g_{2}(0) \alpha_{2}(t)-\frac{\kappa_{i}-\epsilon \kappa_{\mathrm{ex}}}{2} \beta_{\alpha}(t) .
\end{aligned}
$$

The matrix ODE for $\dot{\alpha}_{2}(t)$ and $\dot{\beta}_{\alpha}(t)$ thus takes the following form:

$$
\left(\begin{array}{l}
\dot{\alpha_{2}}(t) \\
\dot{\beta_{\alpha}}(t)
\end{array}\right)=\left(\begin{array}{cc}
-\frac{\Gamma_{i}}{2} & -\frac{1}{\sqrt{1+\epsilon}} g_{2}(0) \\
\sqrt{1+\epsilon} g_{2}(0) & -\frac{\kappa_{\mathrm{ex}}-\epsilon \kappa_{i}}{2}
\end{array}\right)\left(\begin{array}{l}
\alpha_{2}(t) \\
\beta_{\alpha}(t)
\end{array}\right) .
$$

The eigenvalues are similar to those for $t>0$, except for the replacements $\kappa_{\mathrm{ex}} \rightarrow-\epsilon \kappa_{\mathrm{ex}}$ and $g_{1}(0)^{2} \rightarrow g_{2}(0)^{2}$. As such, they can be expressed in terms of a constant $D^{\prime}$ instead of $C$ :

$$
\lambda_{p m}=-\frac{1}{4}\left(\Gamma_{i}-\epsilon \kappa_{\mathrm{ex}}+\kappa_{i}\right) \pm \frac{D^{\prime}}{4},
$$

where $D^{\prime}$ is defined as

$$
D^{\prime}=\sqrt{\left(\Gamma_{i}+\epsilon \kappa_{\mathrm{ex}}-\kappa_{i}\right)^{2}-16 g_{2}(0)^{2}} .
$$

The corresponding eigenvectors vary with $\epsilon$ in the following manner:

$$
v_{ \pm}=\left(\begin{array}{c}
\Gamma_{i}+\epsilon \kappa_{\mathrm{ex}}-\kappa_{i} \mp D^{\prime} \\
-4 g_{2}(0) \sqrt{1+\epsilon}
\end{array}\right) \text {. }
$$

Recall that the solution set for $\alpha_{2}(t)$ and $\beta_{\alpha}(t)$ takes the following generic form:

$$
\left(\begin{array}{l}
\alpha_{2}(t) \\
\beta_{\alpha}(t)
\end{array}\right)=A e^{\lambda_{-} t} v_{-}+B e^{\lambda_{+} t} v_{+}
$$

Since the change in the eigenvalues from the $t>0$ case is fully mediated through the replacements $\kappa_{\mathrm{ex}} \rightarrow-\epsilon \kappa_{\mathrm{ex}}$ and $C \rightarrow D^{\prime}$, the time variance of the individual terms in the solution can also be determined by simply modifying the $t>0$ case by applying the aforementioned replacements, as 
evidenced by expressing the equations in the following form:

$$
\begin{aligned}
\left(\begin{array}{c}
\alpha_{2}(t) \\
\beta_{\alpha}(t)
\end{array}\right) & =e^{-\frac{1}{4}\left(\Gamma_{i}-\epsilon \kappa_{\mathrm{ex}}+\kappa_{i}\right) t}\left(A e^{-\frac{D^{\prime}}{4} t} v_{-}+B e^{\frac{D^{\prime}}{4} t} v_{+}\right) \\
& =e^{-\frac{1}{4}\left(\Gamma_{i}-\epsilon \kappa_{\mathrm{ex}}+\kappa_{i}\right) t}\left[\left(A v_{-}+B v_{+}\right) \cosh \left(\frac{D^{\prime}}{4} t\right)+\left(B v_{+}-A v_{-}\right) \sinh \left(\frac{D^{\prime}}{4} t\right)\right]
\end{aligned}
$$

We now aim to solve the constants $A$ and $B$. The generic matrix ODE solution yields the following system of equations for $\alpha_{2}(0)$ and $\beta_{\alpha}(0)$ in terms of these constants:

$$
\begin{aligned}
& \alpha_{2}(0)=\left(\Gamma_{i}+\epsilon \kappa_{\mathrm{ex}}-\kappa_{i}+D^{\prime}\right) A+\left(\Gamma_{i}+\epsilon \kappa_{\mathrm{ex}}-\kappa_{i}-D^{\prime}\right) B \\
& \beta_{\alpha}(0)=-4 g_{2}(0) \sqrt{1+\epsilon}(A+B) .
\end{aligned}
$$

From this system of equations, we find the following expressions for $A$ and $B$ :

$$
\begin{aligned}
& A=\frac{1}{2 D^{\prime}}\left(\alpha_{2}(0)+\frac{\Gamma_{i}+\epsilon \kappa_{\mathrm{ex}}-\kappa_{i}-D^{\prime}}{4 g_{2}(0) \sqrt{1+\epsilon}} \beta_{\alpha}(0)\right), \\
& B=-\frac{1}{2 D^{\prime}}\left(\alpha_{2}(0)+\frac{\Gamma_{i}+\epsilon \kappa_{\mathrm{ex}}-\kappa_{i}+D^{\prime}}{4 g_{2}(0) \sqrt{1+\epsilon}} \beta_{\alpha}(0)\right) .
\end{aligned}
$$

Next, we use the values of $A, B, v_{+, 1}$, and $v_{-, 1}$ to calculate the constant coefficients in Eq. (C8) for $\alpha_{2}(t)$. Starting with the coefficient in front of $\cosh \left(D^{\prime} t / 4\right)$,

$$
\begin{aligned}
A v_{-, 1}+B v_{+, 1}= & \frac{1}{2 D^{\prime}}\left(\alpha_{2}(0)+\frac{\Gamma_{i}+\epsilon \kappa_{\mathrm{ex}}-\kappa_{i}-D^{\prime}}{4 g_{2}(0) \sqrt{1+\epsilon}} \beta_{\alpha}(0)\right)\left(\Gamma_{i}+\epsilon \kappa_{\mathrm{ex}}-\kappa_{i}+D^{\prime}\right) \\
& -\frac{1}{2 D^{\prime}}\left(\alpha_{2}(0)+\frac{\Gamma_{i}+\epsilon \kappa_{\mathrm{ex}}-\kappa_{i}+D^{\prime}}{4 g_{2}(0) \sqrt{1+\epsilon}} \beta_{\alpha}(0)\right)\left(\Gamma_{i}+\epsilon \kappa_{\mathrm{ex}}-\kappa_{i}-D^{\prime}\right) \\
= & \frac{1}{2 D^{\prime}}\left[2 D^{\prime} \alpha_{2}(0)\right] \\
= & \alpha_{2}(0)
\end{aligned}
$$

For the coefficient in front of $\sinh \left(D^{\prime} t / 4\right)$,

$$
\begin{aligned}
B v_{+, 1}-A v_{-, 1}= & -\frac{1}{2 D^{\prime}}\left(\alpha_{2}(0)+\frac{\Gamma_{i}+\epsilon \kappa_{\mathrm{ex}}-\kappa_{i}+D^{\prime}}{4 g_{2}(0) \sqrt{1+\epsilon}} \beta_{\alpha}(0)\right)\left(\Gamma_{i}+\epsilon \kappa_{\mathrm{ex}}-\kappa_{i}-D^{\prime}\right) \\
& -\frac{1}{2 D^{\prime}}\left(\alpha_{2}(0)+\frac{\Gamma_{i}+\epsilon \kappa_{\mathrm{ex}}-\kappa_{i}-D^{\prime}}{4 g_{2}(0) \sqrt{1+\epsilon}} \beta_{\alpha}(0)\right)\left(\Gamma_{i}+\epsilon \kappa_{\mathrm{ex}}-\kappa_{i}+D^{\prime}\right) \\
= & -\frac{1}{2 D^{\prime}}\left(2\left(\Gamma_{i}+\epsilon \kappa_{\mathrm{ex}}-\kappa_{i}\right) \alpha_{2}(0)+\frac{\left(\Gamma_{i}+\epsilon \kappa_{\mathrm{ex}}-\kappa_{i}\right)^{2}-D^{\prime 2}}{2 g_{2}(0) \sqrt{1+\epsilon}} \beta_{\alpha}(0)\right) \\
= & -\frac{1}{2 D^{\prime}}\left(2\left(\Gamma_{i}+\epsilon \kappa_{\mathrm{ex}}-\kappa_{i}\right) \alpha_{2}(0)+\frac{16 g_{2}(0)^{2}}{2 g_{2}(0) \sqrt{1+\epsilon}} \beta_{\alpha}(0)\right) \\
= & \frac{1}{D^{\prime}}\left(\left(\kappa_{i}-\epsilon \kappa_{\mathrm{ex}}-\Gamma_{i}\right) \alpha_{2}(0)-\frac{4 g_{2}(0)}{\sqrt{1+\epsilon}} \beta_{\alpha}(0)\right) .
\end{aligned}
$$

We substitute these into Eq. (C8) to find $\alpha_{2}(t)$ for $t \leqslant 0$ :

$$
\alpha_{2}(t)=\frac{e^{\frac{1}{4}\left(\epsilon \kappa_{\mathrm{ex}}-\kappa_{i}-\Gamma_{i}\right) t}}{D^{\prime}}\left[\alpha_{2}(0) D^{\prime} \cosh \left(\frac{D^{\prime}}{4} t\right)+\left(-\frac{4 g_{2}(0)}{\sqrt{1+\epsilon}} \beta_{\alpha}(0)+\alpha_{2}(0)\left(-\epsilon \kappa_{\mathrm{ex}}+\kappa_{i}-\Gamma_{i}\right)\right) \sinh \left(\frac{D^{\prime}}{4} t\right)\right]
$$

Note that the constant coefficient in the second term (i.e., the first term in the square brackets) reduces to $-2 \sqrt{2} g_{2}(0) \beta_{\alpha}(0)$ if $\epsilon=1$. 
We now look to solve $\beta_{\alpha}(t)$, starting by substituting the values of $A, B, v_{+, 2}$, and $v_{-, 2}$ to determine the second-row constant coefficients in Eq. (C8). Starting with the coefficient associated with cosh $\left(D^{\prime} t / 4\right)$,

$$
\begin{aligned}
A v_{-, 2}+B v_{+, 2}= & \frac{1}{2 D^{\prime}}\left(\alpha_{2}(0)+\frac{\Gamma_{i}+\epsilon \kappa_{\mathrm{ex}}-\kappa_{i}-D^{\prime}}{4 g_{2}(0) \sqrt{1+\epsilon}} \beta_{\alpha}(0)\right)\left(-4 g_{2}(0) \sqrt{1+\epsilon}\right) \\
& -\frac{1}{2 D^{\prime}}\left(\alpha_{2}(0)+\frac{\Gamma_{i}+\epsilon \kappa_{\mathrm{ex}}-\kappa_{i}+D^{\prime}}{4 g_{2}(0) \sqrt{1+\epsilon}} \beta_{\alpha}(0)\right)\left(-4 g_{2}(0) \sqrt{1+\epsilon}\right) \\
= & \frac{1}{2 D^{\prime}}\left(\frac{-2 D^{\prime}}{4 g_{2}(0) \sqrt{1+\epsilon}} \beta_{\alpha}(0)\right)\left(-4 g_{2}(0) \sqrt{1+\epsilon}\right) \\
= & \beta_{\alpha}(0) .
\end{aligned}
$$

For the coefficient associated with $\sinh \left(D^{\prime} t / 4\right)$,

$$
\begin{aligned}
B v_{+, 2}-A v_{-, 2}= & -\frac{1}{2 D^{\prime}}\left(\alpha_{2}(0)+\frac{\Gamma_{i}+\epsilon \kappa_{\mathrm{ex}}-\kappa_{i}+D^{\prime}}{4 g_{2}(0) \sqrt{1+\epsilon}} \beta_{\alpha}(0)\right)\left(-4 g_{2}(0) \sqrt{1+\epsilon}\right) \\
& -\frac{1}{2 D^{\prime}}\left(\alpha_{2}(0)+\frac{\Gamma_{i}+\epsilon \kappa_{\mathrm{ex}}-\kappa_{i}-D^{\prime}}{4 g_{2}(0) \sqrt{1+\epsilon}} \beta_{\alpha}(0)\right)\left(-4 g_{2}(0) \sqrt{1+\epsilon}\right) \\
= & -\frac{1}{2 D^{\prime}}\left(2 \alpha_{2}(0)+\frac{\Gamma_{i}+\epsilon \kappa_{\mathrm{ex}}-\kappa_{i}}{2 g_{2}(0) \sqrt{1+\epsilon}} \beta_{\alpha}(0)\right)\left(-4 g_{2}(0) \sqrt{1+\epsilon}\right) \\
= & -\frac{1}{D^{\prime}}\left[-4(\sqrt{1+\epsilon}) g_{2}(0) \alpha_{2}(0)+\left(\kappa_{i}-\epsilon \kappa_{\mathrm{ex}}-\Gamma_{i}\right) \beta_{\alpha}(0)\right] .
\end{aligned}
$$

Substituting these into Eq. (C8), we find $\beta_{\alpha}(t)$ for $t \leqslant 0$ :

$$
\beta_{\alpha}(t)=\frac{e^{\frac{1}{4}\left(\epsilon \kappa_{\mathrm{ex}}-\kappa_{i}-\Gamma_{i}\right) t}}{D^{\prime}}\left\{\beta_{\alpha}(0) D^{\prime} \cosh \left(\frac{D^{\prime}}{4} t\right)-\left[-4(\sqrt{1+\epsilon}) g_{2}(0) \alpha_{2}(0)+\beta_{\alpha}(0)\left(-\epsilon \kappa_{\mathrm{ex}}+\kappa_{i}-\Gamma_{i}\right)\right] \sinh \left(\frac{D^{\prime}}{4} t\right)\right\} .
$$

If $\epsilon=1$, the first term in the square brackets reduces to $-4 \sqrt{2} g_{2}(0) \alpha_{2}(0)$.

Finally, we work to solve $\alpha_{1}(t)$ for the first half of the process. Substituting the expression for $g_{1}(t)$ from Eq. (C1) into that for $\dot{\alpha}_{1}(t)$ from Eq. (A26), we obtain the following differential equation:

$$
\begin{aligned}
\dot{\alpha}_{1}(t) & =\frac{\sqrt{\epsilon}}{\sqrt{1+\epsilon}} \frac{\left(-\frac{\kappa_{\mathrm{ex}} \sqrt{\epsilon(1+\epsilon)}}{2} \beta_{\alpha}(t)-\sqrt{\epsilon} g_{2}(0) \alpha_{2}(t)\right)}{\alpha_{1}(t)} \beta_{\alpha}(t)-\frac{\Gamma_{i}}{2} \alpha_{1}(t) \\
& =\frac{\left(-\frac{\kappa_{\mathrm{ex}} \epsilon}{2} \beta_{\alpha}(t)-\frac{\epsilon}{\sqrt{1+\epsilon}} g_{2}(0) \alpha_{2}(t)\right)}{\alpha_{1}(t)} \beta_{\alpha}(t)-\frac{\Gamma_{i}}{2} \alpha_{1}(t) .
\end{aligned}
$$

Multiplying both sides by $2 \alpha_{1}(t)$, the expression becomes a first-order ordinary differential equation in $\alpha_{1}^{2}$ :

$$
\begin{aligned}
\frac{d}{d t} \alpha_{1}(t)^{2} & =2 \alpha_{1}(t) \dot{\alpha}_{1}(t) \\
& =\left(-\kappa_{\mathrm{ex}} \epsilon \beta_{\alpha}(t)^{2}-\frac{2 \epsilon}{\sqrt{1+\epsilon}} g_{2}(0) \alpha_{2}(t) \beta_{\alpha}(t)\right)-\Gamma_{i} \alpha_{1}(t)^{2}
\end{aligned}
$$

As with the other first-order ODE that we have solved [see Eq. (B20)], we integrate this equation term by term:

$$
\alpha_{1}(t)^{2}=e^{-\Gamma_{i} t}\left(-\kappa_{\mathrm{ex}} \epsilon \int e^{\Gamma_{i} t} \beta_{\alpha}(t)^{2} d t-\frac{2 \epsilon}{\sqrt{1+\epsilon}} g_{2}(0) \int e^{\Gamma_{i} t} \alpha_{2}(t) \beta_{\alpha}(t) d t+G^{\prime}\right) .
$$

We observe that $\alpha_{2}(t)$ and $\beta_{\alpha}(t)$ for $t \leqslant 0$ are identical to $\alpha_{1}(t)$ and $\beta_{\alpha}(t)$, respectively, for $t \geqslant 0$ upon making the replacements $C \rightarrow D^{\prime}, \kappa_{\mathrm{ex}} \rightarrow-\epsilon \kappa_{\mathrm{ex}}, \alpha_{1}(0) \rightarrow \alpha_{2}(0), B_{1}^{\prime} \rightarrow B_{3}^{\prime}$, and $B_{2}^{\prime} \rightarrow B_{4}^{\prime}$, where $B_{3}^{\prime}$ and $B_{4}^{\prime}$ represent the bracketed expression in the solution $\beta_{\alpha}(t)$ and the parenthetical expression in the solution $\alpha_{2}(t)$, respectively:

$$
\begin{gathered}
B_{3}^{\prime}=-4(\sqrt{1+\epsilon}) g_{2}(0) \alpha_{2}(0)+\beta_{\alpha}(0)\left(-\epsilon \kappa_{\mathrm{ex}}+\kappa_{i}-\Gamma_{i}\right), \\
B_{4}^{\prime}=-\frac{4 g_{2}(0)}{\sqrt{1+\epsilon}} \beta_{\alpha}(0)+\alpha_{2}(0)\left(-\epsilon \kappa_{\mathrm{ex}}+\kappa_{i}-\Gamma_{i}\right) .
\end{gathered}
$$


We apply the relevant replacements to solve for $\int e^{\Gamma_{i} t} \beta_{\alpha}(t)^{2} d t$ and $\int e^{\Gamma_{i} t} \alpha_{2}(t) \beta_{\alpha}(t) d t$ based on the analogous results for $t \geqslant 0$ from Eqs. (B25) and (B30):

$$
\begin{gathered}
\int e^{\Gamma_{i} t} \beta_{\alpha}(t)^{2} d t=\frac{e^{\frac{1}{2}\left(\Gamma_{i}+\epsilon \kappa_{\mathrm{ex}}-\kappa_{i}\right) t}}{16 g_{2}(0)^{2}}\left[\beta_{\alpha}(0)^{2} A_{4}^{\prime}(t)+2 B_{3}^{\prime} \beta_{\alpha}(0) A_{5}^{\prime}(t)+B_{3}^{\prime 2} A_{6}^{\prime}(t)\right], \\
\int e^{\Gamma_{i} t} \alpha_{1}(t) \beta_{\alpha}(t) d t=\frac{e^{\frac{1}{2}\left(\Gamma_{i}+\epsilon \kappa_{\mathrm{ex}}-\kappa_{i}\right) t}}{16 g_{2}(0)^{2}}\left\{\alpha_{2}(0) \beta_{\alpha}(0) A_{4}^{\prime}(t)+\left[\alpha_{2}(0) B_{3}^{\prime}-\beta_{\alpha}(0) B_{4}^{\prime}\right] A_{5}^{\prime}(t)-B_{3}^{\prime} B_{4}^{\prime} A_{6}(t)\right\},
\end{gathered}
$$

where $A_{4}^{\prime}(t), A_{5}^{\prime}(t)$, and $A_{6}^{\prime}(t)$ are defined by applying the relevant replacements to $A_{1}(t), A_{2}(t)$, and $A_{3}(t)$, respectively:

$$
\begin{aligned}
& A_{4}^{\prime}(t)=\frac{-D^{\prime 2}-D^{\prime}\left(\Gamma_{i}-\kappa_{i}+\epsilon \kappa_{\mathrm{ex}}\right) \sinh \left(\frac{D^{\prime}}{2} t\right)+\left(\Gamma_{i}-\kappa_{i}+\epsilon \kappa_{\mathrm{ex}}\right)^{2}\left[\cosh \left(\frac{D^{\prime}}{2} t\right)+1\right]}{\Gamma_{i}-\kappa_{i}+\epsilon \kappa_{\mathrm{ex}}}, \\
& A_{5}^{\prime}(t)=\frac{-\left(\Gamma_{i}-\kappa_{i}+\epsilon \kappa_{\mathrm{ex}}\right) \sinh \left(\frac{D^{\prime}}{2} t\right)+D^{\prime} \cosh \left(\frac{D^{\prime}}{2} t\right)}{D^{\prime}}, \\
& A_{6}^{\prime}(t)=\frac{D^{\prime 2}-D^{\prime}\left(\Gamma_{i}-\kappa_{i}+\epsilon \kappa_{\mathrm{ex}}\right) \sinh \left(\frac{D^{\prime}}{2} t\right)+\left(\Gamma_{i}-\kappa_{i}+\epsilon \kappa_{\mathrm{ex}}\right)^{2}\left[\cosh \left(\frac{D^{\prime}}{2} t\right)-1\right]}{D^{\prime 2}\left(\Gamma_{i}-\kappa_{i}+\epsilon \kappa_{\mathrm{ex}}\right)} .
\end{aligned}
$$

Substituting the above two integrals into Eq. (C19), we find the following expression for $\alpha_{1}(t)^{2}$ for $t \leqslant 0$ :

$$
\begin{aligned}
\alpha_{1}(t)^{2}= & e^{-\Gamma_{i} t}\left(-\kappa_{\mathrm{ex}} \epsilon \int e^{\Gamma_{i} t} \beta_{\alpha}(t)^{2} d t-\frac{2 \epsilon}{\sqrt{1+\epsilon}} \int e^{\Gamma_{i} t} \alpha_{1}(t) \beta_{\alpha}(t) d t+G^{\prime}\right) \\
= & \frac{e^{-\frac{1}{2}\left(-\epsilon \kappa_{\mathrm{ex}}+\kappa_{i}+\Gamma_{i}\right) t}}{16 g_{2}(0)^{2}}\left\{\left(-\kappa_{\mathrm{ex}} \epsilon \beta_{\alpha}(0)^{2}-\frac{2 \epsilon}{\sqrt{1+\epsilon}} g_{2}(0) \alpha_{2}(0) \beta_{\alpha}(0)\right) A_{4}^{\prime}(t)\right. \\
& +\left(-2 \kappa_{\mathrm{ex}} \epsilon B_{3}^{\prime} \beta_{\alpha}(0)-\frac{2 \epsilon}{\sqrt{1+\epsilon}} g_{2}(0)\left[\alpha_{2}(0) B_{3}^{\prime}-\beta_{\alpha}(0) B_{4}^{\prime}\right]\right) A_{5}^{\prime}(t) \\
& \left.+\left(-\kappa_{\mathrm{ex}} \epsilon B_{3}^{\prime 2}+\frac{2 \epsilon}{\sqrt{1+\epsilon}} g_{2}(0) B_{3}^{\prime} B_{4}^{\prime}\right) A_{6}(t)\right\}+G^{\prime} e^{-\Gamma_{i} t} .
\end{aligned}
$$

Since the system is initially in the $b_{1}$ mode, and since $e^{-\Gamma_{i} t} \approx 1$ for the timescale of the transfer process, $G^{\prime}$ will approximately equal 1.

\section{APPENDIX D: COMPUTATIONAL METHODS}

Having found solutions for the time evolution of the coefficients $\alpha_{1}(t), \alpha_{2}(t)$, and $\beta_{\alpha}(t)$ in terms of their values at $t=0$, our next task is to calculate for the range of possible zero-point values the corresponding coupling profiles $g_{1}(t)$ and $g_{2}(t)$. To this end, we use MATLAB-based computation, setting predetermined values for $g_{1}(0)$ and $g_{2}(0)$. Starting by looping over all possible values of $\alpha_{2}(0)$ and $\beta_{\alpha}(0)$, we substitute these into Eq. (C25) to determine $\alpha_{1}(0)$ for every pair of the input zero-point coefficients. To ensure the validity of the resulting coefficient trios, we only keep the results that closely match the condition in Eq. (A29) for the given values of $g_{1}(0)$ and $g_{2}(0)$. Next, we loop over the filtered list of $t=0$ coefficient trios and substitute them into Eq. (B31) to determine the fidelity $\alpha_{2}\left(t_{f}\right)^{2}$ for each trio. With the consequent knowledge of the time evolution of the coefficients, it is straightforward to use Eq. (B1) to derive $g_{2}(t)$ for $t>0$ and Eq. (C1) to derive $g_{1}(t)$ for $t<0$. The numerical results demonstrate that the fidelity is invariant in the choices of $g_{1}(0)$ and $g_{2}(0)$, while varying specifically with the ratio of intrinsic loss rate to output coupling rate for each of the blocks.
[1] G. Kurizki, P. Bertet, Y. Kubo, K. Molmer, D. Petrosyan, P. Rabl, and J. Schmiedmayer, Quantum technologies with hybrid systems, Proc. Natl. Acad. Sci. U. S. A. 112, 3866 (2015).

[2] G. Wendin, Quantum information processing with superconducting circuits: A review, Rep. Prog. Phys. 80, 106001 (2017).

[3] Y. Chu, P. Kharel, W. H. Renninger, L. D. Burkhart, L. Frunzio, P. T. Rakich, and R. J. Schoelkopf, Quantum acoustics with superconducting qubits, Science 358, 199 (2017).

[4] S. Hong, R. Riedinger, I. Marinkovic, A. Wallucks, S. G. Hofer, R. A. Norte, M. Aspelmeyer, and S. Groblacher, Hanbury Brown and Twiss interferometry of single phonons from an optomechanical resonator, Science 358, 203 (2017).
[5] J. I. Cirac, P. Zoller, H. J. Kimble, and H. Mabuchi, Quantum State Transfer and Entanglement Distribution among Distant Nodes in a Quantum Network, Phys. Rev. Lett. 78, 3221 (1997).

[6] T.-J. Wang, S.-Yu. Song, and G. L. Long, Quantum repeater based on spatial entanglement of photons and quantum-dot spins in optical microcavities, Phys. Rev. A 85, 062311 (2012).

[7] S. Ritter, C. Nölleke, C. Hahn, A. Reiserer, A. Neuzner, M. Uphoff, M. Mücke, E. Figueroa, J. Bochmann, and G. Rempe, An elementary quantum network of single atoms in optical cavities, Nature (London) 484, 195 (2012). 
[8] A. Barfuss, M. Kasperczyk, J. Kölbl, and P. Maletinsky, Spinstress and spin-strain coupling in diamond-based hybrid spin oscillator systems, Phys. Rev. B 99, 174102 (2019).

[9] A. I. Lvovsky, B. C. Sanders, and W. Tittel, Optical quantum memory, Nat. Photonics 3, 706 (2009).

[10] A. H. Safavi-Naeini and O. Painter, Proposal for an optomechanical traveling wave phonon-photon translator, New J. Phys. 13, 013017 (2011).

[11] B. Villalonga, D. Lyakh, S. Boixo, H. Neven, T. S. Humble, R. Biswas, E. G. Rieffel, A. Ho, and S. Mandra, Establishing the quantum supremacy frontier with a 281 Pflop/s simulation, Quantum Sci. Technol. 5, 034003 (2020).

[12] T. E. Northup and R. Blatt, Quantum information transfer using photons, Nat. Photonics 8, 356 (2014).

[13] A. D. Boozer, A. Boca, R. Miller, T. E. Northup, and H. J. Kimble, Reversible State Transfer between Light and a Single Trapped Atom, Phys. Rev. Lett. 98, 193601 (2007).

[14] T. A. Palomaki, J. W. Harlow, J. D. Teufel, R. W. Simmonds, and K. W. Lehnert, Coherent state transfer between itinerant microwave fields and a mechanical oscillator, Nature (London) 495, 210 (2013).

[15] P. Kurpiers, P. Magnard, T. Walter, B. Royer, M. Pechal, J. Heinsoo, Y. Salathé, A. Akin, S. Storz, J.-C. Besse, S. Gasparinetti, A. Blais, and A. Wallraff, Deterministic quantum state transfer and remote entanglement using microwave photons, Nature (London) 558, 264 (2018).

[16] C. J. Axline, L. D. Burkhart, W. Pfaff, M. Zhang, K. Chou, P. Campagne-Ibarcq, P. Reinhold, L. Frunzio, S. M. Girvin, L. Jiang, M. H. Devoret, and R. J. Schoelkopf, On-demand quantum state transfer and entanglement between remote microwave cavity memories, Nat. Phys. 14, 705 (2018).

[17] P.-Y. Hou, Y.-Y. Huang, X.-X. Yuan, X.-Y. Chang, C. Zu, L. He, and L.-M. Duan, Quantum teleportation from light beams to vibrational states of a macroscopic diamond, Nat. Commun. 7, 11736 (2016).

[18] J. Combes, J. Kerckhoff, and M. Sarovar, The SLH framework for modeling quantum input-output networks, Adv. Phys.: X 2 , 784 (2017).

[19] G. Zhang and M. R. James, Direct and indirect couplings in coherent feedback control of linear quantum systems, IEEE Trans. Autom. Control 56, 1535 (2010).

[20] K. Stannigel, P. Komar, S. J. M. Habraken, S. D. Bennett, M. D. Lukin, P. Zoller, and P. Rabl, Optomechanical Quantum Information Processing with Photons and Phonons, Phys. Rev. Lett. 109, 013603 (2012).

[21] S. M. Meenehan, J. D. Cohen, G. S. MacCabe, F. Marsili, M. D. Shaw, and O. Painter, Pulsed Excitation Dynamics of an Optomechanical Crystal Resonator near its Quantum Ground State of Motion, Phys. Rev. X 5, 041002 (2015).

[22] E. Distante, P. Farrera, A. Padron-Brito, D. Paredes-Barato, G. Heinze, and H. de Riedmatten, Storing single photons emitted by a quantum memory on a highly excited Rydberg state, Nat. Commun. 8, 14072 (2017).

[23] Y. Wang, J. Li, S. Zhang, K. Su, Y. Zhou, K. Liao, S. Du, H. Yan, and S.-L. Zhu, Efficient quantum memory for single-photon polarization qubits, Nat. Photonics 13, 346 (2019).
[24] C. W. Gardiner and M. J. Collett, Input and output in damped quantum systems: Quantum stochastic differential equations and the master equation, Phys. Rev. A 31, 3761 (1985).

[25] P. Xu, X.-C. Yang, F. Mei, and Z.-Y. Xue, Controllable highfidelity quantum state transfer and entanglement generation in circuit QED, Sci. Rep. 6, 18695 (2016).

[26] J. R. Johansson, P. D. Nation, and F. Nori, QuTiP: An opensource Python framework for the dynamics of open quantum systems, Comput. Phys. Commun. 183, 1760 (2012).

[27] M. Karuza, M. Galassi, C. Biancofiore, C. Molinelli, R. Natali, P. Tombesi, G. Di Giuseppe, and D. Vitali, Tunable linear and quadratic optomechanical coupling for a tilted membrane within an optical cavity: Theory and experiment, J. Opt. (Bristol) 15, 025704 (2012).

[28] J. D. Thompson, B. M. Zwickl, A. M. Jayich, F. Marquardt, S. M. Girvin, and J. G. E. Harris, Strong dispersive coupling of a high-finesse cavity to a micromechanical membrane, Nature (London) 452, 72 (2008).

[29] N. C. Harris, G. R. Steinbrecher, M. Prabhu, Y. Lahini, J. Mower, D. Bunandar, C. Chen, F. N. C. Wong, T. Baehr-Jones, M. Hochberg, S. Lloyd, and D. Englund, Quantum transport simulations in a programmable nanophotonic processor, Nat. Photonics 11, 447 (2017).

[30] L. Zhuang, C. G. H. Roeloffzen, M. Hoekman, K.-J. Boller, and A. J. Lowery, Programmable photonic signal processor chip for radiofrequency applications, Optica 2, 854 (2015).

[31] J. Han, Z. Li, J. Zhang, H. Xu, K. Linghu, Y. Li, C. Li, M. Chen, Z. Yang, J. Wang, T. Ma, G. Xue, Y. Jin, and H. Yu, Characterizing noise correlation and enhancing coherence via qubit motion, Fundam. Res. 1, 10 (2021).

[32] H. Fan, Coherence enhancement in state transfer with seven-qubit superconducting processor, Fundam. Res. 1, 5 (2021).

[33] X.-S. Xu, H. Zhang, X.-Y. Kong, M. Wang, and G.-L. Long, Frequency-tuning-induced state transfer in optical microcavities, Photonics Res. 8, 490 (2020).

[34] H. Zhou, X. Chen, X. Nie, J. Bian, Y. Ji, Z. Li, and X. Peng, Floquet-engineered quantum state transfer in spin chains, Sci. Bull. 64, 888 (2019).

[35] M.-J. Tao, N.-N. Zhang, P.-Y. Wen, F.-G. Deng, Q. Ai, Z. Li, and G.-L. Long, Coherent and incoherent theories for photosynthetic energy transfer, Sci. Bull. 65, 318 (2019).

[36] Z.-H. Yan, J.-L. Qin, Z.-Z. Qin, X.-L. Su, X.-J. Jia, C.-D. Xie, and K.-C. Peng, Generation of non-classical states of light and their application in deterministic quantum teleportation, Fundam. Res. 1, 43 (2021).

[37] M. Mastriani and S. S. Iyengar, Satellite quantum repeaters for a quantum internet, Quantum Eng. 2, e55 (2020).

[38] G. Y. Wang and G. L. Long, Entanglement purification for memory nodes in a quantum network, Sci. China: Phys., Mech. Astron. 63, 220311 (2020).

[39] B. C. Britt, Modeling viral diffusion using quantum computational network simulation, Quantum Eng. 2, e29 (2020).

[40] F. Sun, Performance analysis of quantum channels, Quantum Eng. 2, e35 (2020). 